Louisiana State University

LSU Digital Commons

$1-1-2017$

\title{
Stability and control design for time-varying systems with time- varying delays using a trajectory-based approach
}

Frederic Mazenc

Laboratoire des Signaux et Systèmes

Michael Malisoff

Louisiana State University

Silviu Iulian Niculescu

Laboratoire des Signaux et Systèmes

Follow this and additional works at: https://digitalcommons.Isu.edu/mathematics_pubs

\section{Recommended Citation}

Mazenc, F., Malisoff, M., \& Niculescu, S. (2017). Stability and control design for time-varying systems with time-varying delays using a trajectory-based approach. SIAM Journal on Control and Optimization, 55 (1), 533-556. https://doi.org/10.1137/15M1027838

This Article is brought to you for free and open access by the Department of Mathematics at LSU Digital Commons. It has been accepted for inclusion in Faculty Publications by an authorized administrator of LSU Digital Commons. For more information, please contact ir@lsu.edu. 
archives-ouvertes

\section{Stability and Control Design for Time-Varying Systems with Time-Varying Delays using a Trajectory-Based Approach}

Frédéric Mazenc, Michael Malisoff, Silviu-Iulian Niculescu

\section{To cite this version:}

Frédéric Mazenc, Michael Malisoff, Silviu-Iulian Niculescu. Stability and Control Design for TimeVarying Systems with Time-Varying Delays using a Trajectory-Based Approach. SIAM Journal on Control and Optimization, Society for Industrial and Applied Mathematics, 2017, 55 (1), pp.533 556. 10.1137/15M1027838 . hal-01660100

\section{HAL Id: hal-01660100 https://hal.inria.fr/hal-01660100}

Submitted on 11 Dec 2017

HAL is a multi-disciplinary open access archive for the deposit and dissemination of scientific research documents, whether they are published or not. The documents may come from teaching and research institutions in France or abroad, or from public or private research centers.
L'archive ouverte pluridisciplinaire HAL, est destinée au dépôt et à la diffusion de documents scientifiques de niveau recherche, publiés ou non, émanant des établissements d'enseignement et de recherche français ou étrangers, des laboratoires publics ou privés. 


\title{
Stability and Control Design for Time-Varying Systems with Time-Varying Delays using a Trajectory Based Approach*
}

\author{
Frederic Mazenc $^{\dagger} \quad$ Michael Malisoff ${ }^{\ddagger} \quad$ Silviu-Iulian Niculescu ${ }^{\dagger}$
}

\begin{abstract}
A recent work by Mazenc and Malisoff provides a trajectory-based approach for proving stability of time-varying systems with time-varying delays. Here, we provide several significant applications of their approach. In two results, we use a Lyapunov function for a corresponding undelayed system to provide a new method for proving stability of linear continuous-time time-varying systems with bounded timevarying delays. We allow uncertainties in the coefficient matrices of the systems. Our main results use upper bounds on an integral average involving the delay. The results establish input-to-state stability with respect to disturbances. We also provide a novel reduction model approach that ensures global exponential stabilization of linear systems with a time-varying pointwise delay in the input, which allows the delay to be discontinuous and uncertain. Finally, we provide an alternative to the reduction model method, based on a different dynamic extension. Our examples demonstrate the usefulness of our findings in several settings.
\end{abstract}

Key Words: delay, reduction approach, time-varying, stability

\section{Introduction}

Delays occur in many engineering applications [6, 11, 44, 45], where it may not be feasible to continuously measure the current state of the system. Stability analysis for systems with time-varying delays is significant from the applied and theoretical viewpoints, especially when the delays have discontinuities. Motivations for nonconstant delays occur in networked systems, and systems with nonconstant delays have been widely studied; see $[2,9,10,15,26,28,42,47,49]$ and [17, Chapt. 6]. Systems with nonconstant delays are challenging, since in most cases, standard frequency-domain and Lyapunov-Krasovskii methods are not generally applicable.

We can use time-varying delays $h(t)$ to model many situations, such as sampling in controllers [14] (where $\dot{h}(t)=1$ almost everywhere, so the delay has jump discontinuities at each sampling time) and delayed muscle response due to fatigue [11] (where $h$ may increase). Moreover, the upper bound on the time-varying delays that preserve stability may exceed the corresponding bound for constant delays; see [30], as well as the discussion [31] of quenching. Also, even if a dynamics of the form $\dot{x}(t)=\mathcal{F}(x(t), x(t-\tau))$ is exponentially stable for all constant delays $\tau$ on a given interval $\left[0, \tau_{\max }\right]$, it may not necessarily be true that $\dot{x}(t)=\mathcal{F}(x(t), x(t-h(t)))$ is exponentially stable for all nonconstant delays $h:[0, \infty) \rightarrow\left[0, \tau_{\max }\right]$; see the Markus-Yanabe phenomenon [18], [20], [33]. There may be other types of time-varying delays, such as distributed delays. This makes the study of systems with nonconstant delays an important problem in control theory.

The study of systems with time-varying delays is often complicated by the fact that the systems may be time-varying when the delay is zero [17]. Time-varying systems often arise in tracking problems, even if we use a linear approximation (since the coefficient matrices in the linearization will be time-varying) [39]. It is common in the literature to require the largest possible delay value to be sufficiently small [38], or that certain matrices associated with the vector field are confined to certain compact sets [17, Chapt. 6]. Another standard

\footnotetext{
${ }^{*}$ Corresponding author: F. Mazenc. A preliminary version appeared in the proceedings of the 54th IEEE Conference on Decision and Control; see the end of Section I for the differences between the conference version and this paper.

${ }^{\dagger}$ F. Mazenc and S-I. Niculescu are with EPI DISCO INRIA-Saclay, Laboratoire des Signaux et Systèmes, CNRS, CentraleSupélec, Université Paris-Sud, 3 rue Joliot Curie, 91192, Gif-sur-Yvette, France. frederic.mazenc@12s.centralesupelec.fr and Silviu.NICULESCU@lss.supelec.fr. The work of F. Mazenc was partly supported by l'Institut pour le Contrôle et la Décision de l'Idex Paris-Saclay (iCODE).

${ }^{\ddagger}$ M. Malisoff is with Department of Mathematics, Louisiana State University (LSU), 303 Lockett Hall, Baton Rouge, LA 708034918, malisoff@lsu.edu. Supported by NSF-ECCS Grants 1102348 and 1408295 and Roy P. Daniels Professorship \#3 at LSU.
} 
assumption on the delay $h(t)$ is that $\phi(t)=t-h(t)$ is invertible [28]. The work [9] is an exception. In [9], an averaging assumption on an integral of $|\dot{h}|^{2}$ is imposed, instead of assuming that $\phi(t)=t-h(t)$ is invertible.

The present paper has connections with [9], since our assumptions also involve integrals that incorporate the delays $h$. However, our main results do not require the differentiability of the delay function $h$, and [9] is confined to constant coefficient linear systems with predictive controls. To cope with the time-varying character of the systems as well as the time-varying delay, we apply a trajectory-based approach from [35]. In our opinion, two potential advantages of the approach from [35] are that it does not require the Lyapunov or small gain conditions that are common in the literature, and that it does not restrict the upper bounds on $h(t)$. We are unaware of any other method to prove the results in the present paper. The size restrictions on $h(t)$ from [16] and [37] are not in general satisfied by our systems. Furthermore, we also do not need the time-varying matrices involving the vector fields to belong to specific compact sets, as was imposed in [17, Chapt. 6]. Unlike [9], we cover linear time-varying systems with a time-varying piecewise continuous delay $h(t)$. The assumptions from [28] may be violated by our systems, and our results imply exponential stability.

Two of our theorems here are inspired by emulation, insofar that they place assumptions on corresponding undelayed systems, and then they study the effects of the delays. Emulation is generally a two step process. In the first step, one solves a stabilization problem with the input delays set to zero, by constructing a Lyapunov function for the closed-loop undelayed system and then computing decay estimates for the Lyapunov function. In the second step, one reintroduces the input delays and converts the Lyapunov function into a LyapunovKrasovskii functional for the corresponding input delayed systems, in order to find upper bounds on the input delays that the system can tolerate while maintaining the stability in closed loop. A potential advantage of emulation is that it makes it possible to use relatively simple controllers, but emulation cannot always cover communications and other engineering applications where the delays are long relative to the total response time of the system [16].

In such cases reduction appears as a useful alternative, in which the controller involves a dynamic extension, and can often compensate for arbitrarily long input delays $[1,7,12,29,41]$. Although it has its origins in the Smith predictor [48] for linear systems (hence the term prediction $[22,28]$ ), recent prediction results apply to many other systems, including perturbed dynamics $[2-5,8,27]$. The work [9] used a transport PDE, reduction, and a generalized Halanay inequality $[19,21]$ to prove stability of linear time invariant systems with known input delays $h(t)$ that can satisfy $\dot{h}(t)>1$ for some $t$ 's. This sets [9] apart from the usual results that require $\sup _{t} \dot{h}(t) \leq 1$, so [9] is a significant advance in the use of reduction to prove stability of systems with time-varying delays. One of the key assumptions in [9] is that $|\dot{h}|^{2}$ is small in a suitable averaged sense, without requiring a bound on $|\dot{h}|$. This makes it possible to use [9] to cover chattering in delays that occur in many engineering systems.

In our third and fourth theorems, we pursue a related line of research involving dynamic extensions for time-varying systems with time-varying delays, but our results differ from [9] in several key ways. First, we allow the delays to contain discontinuities and uncertainties. Also, we use a very different set of assumptions from [9], which allow us to prove stability using our trajectory approach from [35] instead of using transport PDEs. It is important to point out that the trajectory based method from [35] was not used in [9]. We also provide an alternative approach to dynamic controls under time-varying delays, using a dynamic extension from $[32,43]$ from the theory of spectrum assignment. In all of our results in this paper, we allow time-varying linear systems with additive uncertainty in the coefficient matrices, which can capture important cases where the system arises from linearizing around a desired reference trajectory, but where the reference trajectory itself is subject to uncertainties.

Our conference papers $[36,40]$ presented special cases of this work in which there is no uncertainty in the coefficient matrices, and they did not include the proofs detailed below. By covering linear time-varying systems with uncertain coefficients and providing proofs, this work adds considerable value relative to [36, 40].

\section{Definitions, Notation, and Prerequisites}

Throughout this paper, all dimensions are arbitrary, unless indicated otherwise. The usual Euclidean norm, and its induced matrix norm, are denoted by $|\cdot|,|\cdot|_{\mathcal{I}}$ denotes the (essential) supremum over any interval $\mathcal{I} \subseteq \mathbb{R}$, and $I_{r}$ is the identity matrix in dimension $r$. Let $\lambda_{\max }(Q)>0\left(\right.$ resp., $\lambda_{\min }(Q)>0$ ) be the biggest (resp., smallest) eigenvalue of any positive definite symmetric matrix $Q$, and $C^{1}$ be the set of all continuously 
differentiable functions, whose domains and ranges will be clear from the context. For any matrices $P$ and $Q$ in $\mathbb{R}^{n \times n}$, we use $P<Q$ (resp., $\left.P \leq Q\right)$ to mean that $Q-P$ is positive definite (resp., positive semidefinite). For each constant delay bound $\tau>0$, let $C\left([-\tau, 0], \mathbb{R}^{n}\right)$ be the set of all continuous $\mathbb{R}^{n}$-valued functions defined on $[-\tau, 0]$. We let $C_{\text {in }}$ be the set of all absolutely continuous functions $\phi \in C\left([-\tau, 0], \mathbb{R}^{n}\right)$, which we call the set of all initial functions. For each continuous function $\varphi:[-\tau, \infty) \rightarrow \mathbb{R}^{n}$ and all $t \geq 0$, we set $\varphi_{t}(m)=\varphi(t+m)$ for all $m \in[-\tau, 0]$. A function defined on an interval $\mathcal{I} \subseteq \mathbb{R}$ is called piecewise continuous provided it is continuous at all points of $\mathcal{I}$ except at finitely many points on each bounded subinterval of $\mathcal{I}$ (which includes continuous functions as a special case). When referring to a function $\phi$ that is defined on $[0, \infty)$ as being differentiable, we view $\phi^{\prime}(0)$ as being the right derivative at 0 .

Let $\mathcal{K}$ denote the set of all strictly increasing continuous functions $\alpha:[0, \infty) \rightarrow[0, \infty)$ such that $\alpha(0)=0$; if, in addition, $\alpha$ is unbounded, then we say that $\alpha$ is of class $\mathcal{K}_{\infty}$. We say that a continuous function $\beta$ : $[0, \infty) \times[0, \infty) \rightarrow[0, \infty)$ is of class $\mathcal{K} \mathcal{L}$ provided (a) for each $s \geq 0$, the function $\beta(\cdot, s)$ is in $\mathcal{K}$ and (b) for each choice of $r \geq 0$, the function $\beta(r, \cdot)$ is non-increasing and satisfies $\lim _{s \rightarrow \infty} \beta(r, s)=0$. For simplicity, we assume that all of our initial times for our initial value problems are 0 , but more general cases can be covered by similar arguments. We say that a system of the form $\dot{x}(t)=f(t, x(t), u(t-h(t)), \varepsilon(t))$, having a time delay $h(t)$ that admits a constant $\tau>0$ such that $0 \leq h(t) \leq \tau$ for all $t \geq 0$, is input-to-state stable (or ISS) [25] with respect to $\varepsilon:[0, \infty) \rightarrow \mathbb{R}^{m}$ provided the following condition holds: There exist functions $\beta \in \mathcal{K} \mathcal{L}$ and $\gamma \in \mathcal{K}_{\infty}$ such that for all initial functions and all locally bounded piecewise continuous functions $\varepsilon:[0, \infty) \rightarrow \mathbb{R}^{m}$, the corresponding solution $\sigma_{f}(t)=\phi\left(t, x_{0}, \varepsilon\right)$ of the system is defined on $[-\tau, \infty)$ and satisfies $\left|\phi\left(t, x_{0}, \varepsilon\right)\right| \leq \beta\left(\left|x_{0}\right|_{[-\tau, 0]}, t\right)+\gamma\left(|\varepsilon|_{[0, t]}\right)$ for all $t \geq 0$. This reduces to the standard global exponential stability (or GES) condition when the perturbations $\varepsilon$ are all zero and $\beta(s, t)=c_{1} s e^{-c_{2} t}$ for some constants $c_{1}>0$ and $c_{2}>0$. We say that a function $\mathcal{P}: \mathbb{R} \times C_{\mathrm{in}} \rightarrow C_{\mathrm{in}}$ is locally Lipschitz in its second argument provided that for each $\phi \in C_{\text {in }}$, there exist constants $r>0$ and $K_{r}>0$ such that for all $\phi_{1} \in C_{\text {in }}$ and $\phi_{2} \in C_{\text {in }}$ such that $\left|\phi_{1}-\phi\right|_{[-\tau, 0]} \leq r$ and $\left|\phi_{2}-\phi\right|_{[-\tau, 0]} \leq r$, and for all $t \in \mathbb{R}$, we have $\left|\mathcal{P}\left(t, \phi_{1}\right)-\mathcal{P}\left(t, \phi_{2}\right)\right|_{[-\tau, 0]} \leq K_{r}\left|\phi_{1}-\phi_{2}\right|_{[-\tau, 0]}$.

\section{Key Preliminary Results}

This section provides three lemmas and a theorem that are key for proving the stability results in the next sections; see (36), (69), and (83) for the classes of systems that we will later cover by our theory. We first establish a slightly less restrictive version of the trajectory based result from [35, Lemma 1]; it differs from [35, Lemma 1] because the offset term in [35, Lemma 1] was $|d|_{[0, t]} /(1-\rho)^{2}$ while the offset in our new version is $|d|_{[0, t]} /(1-\rho)$. Since $\rho \in(0,1)$, our new lemma therefore provides a tighter estimate.

Lemma 1. Let $T_{*}>0$ be a constant. Let $w:\left[-T_{*}, \infty\right) \rightarrow[0, \infty)$ be a piecewise continuous locally bounded function and $d:[0, \infty) \rightarrow[0, \infty)$ be piecewise continuous. Assume that there exists a constant $\rho \in(0,1)$ such that

$$
w(t) \leq \rho|w|_{\left[t-T_{*}, t\right]}+d(t)
$$

holds for all $t \geq 0$. Then the inequality

$$
w(t) \leq|w|_{\left[-T_{*}, 0\right]} e^{\frac{\ln (\rho)}{T_{*}} t}+\frac{1}{1-\rho}|d|_{[0, t]}
$$

holds for all $t \geq 0$.

Proof. Set $d_{b}(t)=|d|_{[0, t]}$ for all $t \geq 0$ and $\alpha(t)=d_{b}(\max \{0, t\}) /(1-\rho)$ for all $t \geq-T_{*}$. Then, (1) implies that

$$
w(t) \leq \rho|w|_{\left[t-T_{*}, t\right]}+d_{b}(t)
$$

for all $t \geq 0$. Since $w$ is nonnegative valued and $\alpha$ is nondecreasing, this leads to

$$
w(t)-\alpha(t) \leq \rho|w|_{\left[t-T_{*}, t\right]}+d_{b}(t)-\alpha(t)=\rho\left(|w|_{\left[t-T_{*}, t\right]}-\alpha(t)\right) \leq \rho \sup _{\ell \in\left[t-T_{*}, t\right]}(w(\ell)-\alpha(\ell))
$$

for all $t \geq 0$. Let $t \geq 0$ and $\Omega(t)=\max \{w(t)-\alpha(t), 0\}$. If $w(t) \leq \alpha(t)$, then $\Omega(t)=0$, which implies that

$$
\Omega(t) \leq \rho|\Omega|_{\left[t-T_{*}, t\right]} .
$$


On the other hand, if $w(t)>\alpha(t)$, then (3) leads to $0<\Omega(t)=w(t)-\alpha(t) \leq \rho \sup _{\ell \in\left[t-T_{*}, t\right]}(w(\ell)-\alpha(\ell))$, in which case $\sup _{\ell \in\left[t-T_{*}, t\right]}(w(\ell)-\alpha(\ell))>0$, which implies that $\sup _{\ell \in\left[t-T_{*}, t\right]}(w(\ell)-\alpha(\ell))=|\Omega|_{\left[t-T_{*}, t\right]}$. Consequently, (4) holds in both cases. It therefore follows from applying [35, Lemma 1] to the function $\Omega$ that

$$
\max \{w(t)-\alpha(t), 0\}=\Omega(t) \leq|\Omega|_{\left[-T_{*}, 0\right]} e^{\frac{\ln (\rho)}{T_{*}} t}=|\max \{w-\alpha, 0\}|_{\left[-T_{*}, 0\right]} e^{\frac{\ln (\rho)}{T_{*}} t}
$$

holds for all $t \geq 0$.

We now consider two cases. If $t \geq 0$ is such that $w(t)>\alpha(t)$, then (5) leads to

$$
0<w(t)-\alpha(t) \leq|\max \{w-\alpha, 0\}|_{\left[-T_{*}, 0\right]} e^{\frac{\ln (\rho)}{T_{*}} t} .
$$

It follows that, in this case, we have the following:

$$
0<|\max \{w-\alpha, 0\}|_{\left[-T_{*}, 0\right]}=\sup \left\{w(m)-\alpha(m): m \in\left[-T_{*}, 0\right], w(m)>\alpha(m)\right\} \leq|w|_{\left[-T_{*}, 0\right]}
$$

and therefore (6) gives

$$
w(t) \leq|w|_{\left[-T_{*}, 0\right]} e^{\frac{\ln (\rho)}{T_{*}} t}+\alpha(t) .
$$

On the other hand, if $t \geq 0$ is such that $w(t) \leq \alpha(t)$, then (8) holds again, since the first right side term in (8) is nonnegative, so (8) holds in both cases. This proves the lemma.

The next lemma differs from a Lyapunov type argument because the function $p$ is allowed to take negative values, which makes it very different from a contractiveness lemma.

Lemma 2. Let $Z: \mathbb{R} \rightarrow[0, \infty), R: \mathbb{R} \rightarrow[0, \infty)$ and $p: \mathbb{R} \rightarrow \mathbb{R}$ be functions such that $Z$ is $C^{1}, p$ is piecewise continuous and locally bounded, $R$ is locally integrable, and

$$
\dot{Z}(t) \leq-p(t) Z(t)+\sqrt{Z(t)} R(t)
$$

holds for all $t \geq 0$. Then, for all nonnegative values $s$ and $t$ such that $s \leq t$, the inequality

$$
\sqrt{Z(t)} \leq e^{-\frac{1}{2} \int_{s}^{t} p(m) \mathrm{d} m} \sqrt{Z(s)}+\frac{1}{2} \int_{s}^{t} e^{-\frac{1}{2} \int_{\ell}^{t} p(m) \mathrm{d} m} R(\ell) \mathrm{d} \ell
$$

holds.

Proof. Let $\epsilon>0$ be a constant and $Y_{\epsilon}(\ell)=\sqrt{Z(\ell)+\epsilon}-\sqrt{\epsilon}$. Since $R$ and $Z$ are nonnegative valued, (9) leads to

$$
\begin{aligned}
\dot{Y}_{\epsilon}(\ell) & \leq-p(\ell) \frac{Z(\ell)}{2 \sqrt{Z(\ell)+\epsilon}}+\frac{\sqrt{Z(\ell)}}{2 \sqrt{Z(\ell)+\epsilon}} R(\ell) \\
& \leq-\frac{p(\ell)}{2} Y_{\epsilon}(\ell)+\frac{1}{2} R(\ell)+\frac{p(\ell)}{2}\left[Y_{\epsilon}(\ell)+\frac{\epsilon-\left(Y_{\epsilon}(\ell)+\sqrt{\epsilon}\right)^{2}}{Y_{\epsilon}(\ell)+\sqrt{\epsilon}}\right]=-\frac{p(\ell)}{2} Y_{\epsilon}(\ell)+\frac{1}{2} R(\ell)-\frac{\sqrt{\epsilon} p(\ell)}{2\left(Y_{\epsilon}(\ell)+\sqrt{\epsilon}\right)} Y_{\epsilon}(\ell)
\end{aligned}
$$

for all $\ell \in[s, t]$. By integrating (11) on $[s, t]$, and then substituting in the definition of $Y_{\epsilon}$, we obtain:

$$
\begin{aligned}
\sqrt{Z(t)+\epsilon}-\sqrt{\epsilon} \leq & e^{-\frac{1}{2} \int_{s}^{t} p(m) \mathrm{d} m}(\sqrt{Z(s)+\epsilon}-\sqrt{\epsilon})+\int_{s}^{t} e^{-\frac{1}{2} \int_{\ell}^{t} p(m) \mathrm{d} m} \frac{1}{2} R(\ell) \mathrm{d} \ell \\
& -\sqrt{\epsilon} \int_{s}^{t} p(\ell) e^{-\frac{1}{2} \int_{\ell}^{t} p(m) \mathrm{d} m}\left\{\frac{1}{2 \sqrt{Z(\ell)+\epsilon}}(\sqrt{Z(\ell)+\epsilon}-\sqrt{\epsilon})\right\} \mathrm{d} \ell .
\end{aligned}
$$

Since (12) is valid for all $\epsilon>0$, and since the quantity in curly braces in (12) is bounded by 1 , the inequality (12) is true for $\epsilon=0$. This proves the lemma.

We introduced $\epsilon>0$ in the previous proof because it makes it possible to establish (10) with functions that are of class $C^{1}$ everywhere. By contrast, if we had set $\epsilon=0$ throughout the proof, then we would have needed to study the non-differentiable function $Y_{0}$. The next lemma involves a decay condition that is reminiscent of a Riccati inequality, but is different since the function $p(t)$ in the lemma can again take positive and negative values. 
Lemma 3. Consider the system

$$
\dot{X}(t)=\mathcal{F}(t) X(t)+\mathcal{M}\left(t, X_{t}\right),
$$

where $X$ is valued in $\mathbb{R}^{n}, \mathcal{F}:[0, \infty) \rightarrow \mathbb{R}^{n \times n}$ is piecewise continuous and locally bounded, and $\mathcal{M}:[0, \infty) \times C_{\text {in }} \rightarrow$ $\mathbb{R}^{n}$ is locally integrable in its first argument and locally Lipschitz in its other argument. Assume that there is a $C^{1}$ function $\mathcal{P}:[0, \infty) \rightarrow \mathbb{R}^{n \times n}$ such that $\mathcal{P}$ is symmetric and positive definite for all $t \geq 0$, constants $p_{s}$ and $p_{l}$ such that $p_{l} \geq p_{s}>0$, and a continuous function $p:[0, \infty) \rightarrow \mathbb{R}$ such that

$$
\dot{\mathcal{P}}(t)+\mathcal{F}(t)^{\top} \mathcal{P}(t)+\mathcal{P}(t) \mathcal{F}(t) \leq-p(t) \mathcal{P}(t) \quad \text { and } \quad p_{s} I_{n} \leq \mathcal{P}(t) \leq p_{l} I_{n}
$$

hold for all $t \geq 0$. Then

$$
|X(t)| \leq \sqrt{\frac{p_{l}}{p_{s}}} e^{-\frac{1}{2} \int_{r}^{t} p(m) \mathrm{d} m}|X(r)|+\sqrt{\frac{p_{l}}{p_{s}}} \int_{r}^{t} e^{-\frac{1}{2} \int_{\ell}^{t} p(m) \mathrm{d} m}\left|\mathcal{M}\left(\ell, X_{\ell}\right)\right| \mathrm{d} \ell
$$

holds for all $t$ and $r$ such that $t \geq r \geq 0$ and for all solutions of (13).

Proof. Let $\nu(t, X)=X^{\top} \mathcal{P}(t) X$. For each $t \geq 0$, let $\mathcal{P}(t)^{1 / 2}$ be a symmetric positive definite matrix such that $\mathcal{P}(t)=\mathcal{P}(t)^{1 / 2} \mathcal{P}(t)^{1 / 2}$. Then $\sqrt{\nu(t, X)}=\left|X^{\top} \mathcal{P}(t)^{1 / 2}\right|$ and $p_{s}|X|^{2} \leq \nu(t, X) \leq p_{l}|X|^{2}$ hold for all $t \geq 0$ and $X \in \mathbb{R}^{n}$. From (14), it follows that the time-derivative of $\nu$ along the trajectories of (13) satisfies:

$$
\dot{\nu}(t) \leq-p(t) \nu(t, X(t))+2 \sqrt{\nu(t, X(t))}\left|\mathcal{P}(t)^{1 / 2} \mathcal{M}\left(t, X_{t}\right)\right| .
$$

We now apply Lemma 2 , with the choices $Z(t)=\nu(t, X(t))$ and $R(t)=2\left|\mathcal{P}(t)^{1 / 2} \mathcal{M}\left(t, X_{t}\right)\right|$. It follows that for all $r \geq 0$ and $t \geq r$,

$$
\sqrt{\nu(t, X(t))} \leq e^{-\frac{1}{2} \int_{r}^{t} p(m) \mathrm{d} m} \sqrt{\nu(r, X(r))}+\int_{r}^{t} e^{-\frac{1}{2} \int_{\ell}^{t} p(m) \mathrm{d} m}\left|\mathcal{P}(\ell)^{1 / 2} \mathcal{M}\left(\ell, X_{\ell}\right)\right| \mathrm{d} \ell
$$

holds. By our bounds $p_{s}|X|^{2} \leq \nu(t, X) \leq p_{l}|X|^{2}$, (17) leads to

$$
|X(t)| \leq \sqrt{\frac{p_{l}}{p_{s}}} e^{-\frac{1}{2} \int_{r}^{t} p(m) \mathrm{d} m}|X(r)|+\frac{1}{\sqrt{p_{s}}} \int_{r}^{t} e^{-\frac{1}{2} \int_{\ell}^{t} p(m) \mathrm{d} m}\left|\mathcal{P}(\ell)^{1 / 2} \mathcal{M}\left(\ell, X_{\ell}\right)\right| \mathrm{d} \ell .
$$

Since $\left|\mathcal{P}(t)^{1 / 2}\right| \leq \sqrt{p_{l}}$ holds for all $t \geq 0$, we conclude that (15) is satisfied.

The next result is important for proving the results in the next sections on time-varying linear systems with perturbations and delays. See Remark 1 for a way to convert its conclusion into an ISS estimate.

Theorem 1. Let $g>0$ be any constant. Consider any system of the form

$$
\dot{X}(t)=\mathcal{F}(t) X(t)+\mathcal{U}\left(t, X_{t}\right)
$$

with initial conditions in $C\left([-g, 0], \mathbb{R}^{n}\right)$, where $\mathcal{F}:[0, \infty) \rightarrow \mathbb{R}^{n \times n}$ is piecewise continuous and locally bounded, and $\mathcal{U}$ is locally integrable in its first argument $t$ and locally Lipschitz in its second argument. Assume that there are locally bounded piecewise continuous functions $\lambda:[0, \infty) \rightarrow[0, \infty)$ and $\mu:[0, \infty) \rightarrow[0, \infty)$ such that

$$
|\mathcal{U}(t, \phi)| \leq \mu(t) \sup _{m \in[-g, 0]}|\phi(m)|+\lambda(t)
$$

holds for all $t \geq 0$ and all $\phi \in C_{\mathrm{in}}$. Finally, assume there exist a $C^{1}$ function $\mathcal{P}:[0, \infty) \rightarrow \mathbb{R}^{n}$ such that $\mathcal{P}(t)$ is symmetric and positive definite for all $t \geq 0$; constants $p_{*}>0, T \geq g$, and $\delta \in(0,1)$; a continuous function $p:[0, \infty) \rightarrow\left[-p_{*}, \infty\right) ;$ and two constants $p_{s}$ and $p_{l}$ such that $p_{l} \geq p_{s}>0$ and such that

$$
\dot{\mathcal{P}}(t)+\mathcal{F}(t)^{\top} \mathcal{P}(t)+\mathcal{P}(t) \mathcal{F}(t) \leq-p(t) \mathcal{P}(t) \text { and } p_{s} I_{n} \leq \mathcal{P}(t) \leq p_{l} I_{n}
$$

hold for all $t \geq 0$ and such that

$$
\sqrt{\frac{p_{l}}{p_{s}}}\left[e^{-\frac{1}{2} \int_{t-T}^{t} p(m) \mathrm{d} m}+\int_{t-T}^{t} e^{-\frac{1}{2} \int_{\ell}^{t} p(m) \mathrm{d} m} \mu(\ell) \mathrm{d} \ell\right] \leq \delta
$$

holds for all $t \geq T$. Then for all $t \geq T$, the inequality

$$
|X(t)| \leq e^{\frac{\ln (\delta)}{T+g}(t-T)}|X|_{[-g, T]}+\frac{2 \sqrt{p_{l}}\left(e^{T p_{*} / 2}-1\right)}{p_{*}(1-\delta) \sqrt{p_{s}}}|\lambda|_{[0, t]}
$$

is satisfied along all solutions of (19). 
Proof. By (21), we deduce that Lemma 3 applies, with $\mathcal{M}=\mathcal{U}$. Hence, for all $t$ and $r$ such that $0 \leq r \leq t$,

$$
|X(t)| \leq \sqrt{\frac{p_{l}}{p_{s}}} e^{-\frac{1}{2} \int_{r}^{t} p(m) \mathrm{d} m}|X(r)|+\sqrt{\frac{p_{l}}{p_{s}}} \int_{r}^{t} e^{-\frac{1}{2} \int_{\ell}^{t} p(m) \mathrm{d} m}\left|\mathcal{U}\left(\ell, X_{\ell}\right)\right| \mathrm{d} \ell
$$

is satisfied. Using (20), it follows that if $0 \leq r \leq t$, then

$$
\begin{aligned}
|X(t)| & \leq \sqrt{\frac{p_{l}}{p_{s}}} e^{-\frac{1}{2} \int_{r}^{t} p(m) \mathrm{d} m}|X(r)|+\sqrt{\frac{p_{l}}{p_{s}}} \int_{r}^{t} e^{-\frac{1}{2} \int_{\ell}^{t} p(m) \mathrm{d} m}\left(\mu(\ell) \sup _{m \in[\ell-g, \ell]}|X(m)|+\lambda(\ell)\right) \mathrm{d} \ell \\
& \leq \sqrt{\frac{p_{l}}{p_{s}}}\left[e^{-\frac{1}{2} \int_{r}^{t} p(m) \mathrm{d} m}+\int_{r}^{t} e^{-\frac{1}{2} \int_{\ell}^{t} p(m) \mathrm{d} m} \mu(\ell) \mathrm{d} \ell\right]|X|_{[r-g, t]}+\sqrt{\frac{p_{l}}{p_{s}}} \int_{r}^{t} e^{-\frac{1}{2} \int_{\ell}^{t} p(m) \mathrm{d} m} \lambda(\ell) \mathrm{d} \ell .
\end{aligned}
$$

It follows from (22), and from choosing $r=t-T$ in (25), that for all $t \geq T$, we have the following:

$$
|X(t)| \leq \delta|X|_{[t-T-g, t]}+\sqrt{\frac{p_{l}}{p_{s}}} \int_{t-T}^{t} e^{-\frac{1}{2} \int_{\ell}^{t} p(m) \mathrm{d} m} \lambda(\ell) \mathrm{d} \ell .
$$

Let $\mathcal{S}(\ell)=|X(\ell+T)|$ for all $\ell \geq 0$. By setting $t=\ell+T$ in (26), it follows that for all $\ell \geq 0$, we have

$$
\mathcal{S}(\ell) \leq \delta \mathcal{S}_{[\ell-T-g, \ell]}+\sqrt{\frac{p_{l}}{p_{s}}} \int_{\ell}^{\ell+T} e^{-\frac{1}{2} \int_{q}^{\ell+T} p(a) \mathrm{d} a} \lambda(q) \mathrm{d} q .
$$

Since $\delta \in(0,1)$, we deduce from Lemma 1 with $T_{*}=T+g$ that for all $\ell \geq 0$, we have

$$
\mathcal{S}(\ell) \leq \mathcal{S}_{[-T-g, 0]} e^{\frac{\ln (\delta)}{T+g} \ell}+\frac{1}{1-\delta} \sup _{u \in[0, \ell]} \sqrt{\frac{p_{l}}{p_{s}}} \int_{u}^{u+T} e^{-\frac{1}{2} \int_{q}^{u+T} p(a) \mathrm{d} a} \lambda(q) \mathrm{d} q .
$$

Consequently, by setting $\ell=t-T$ in (28), it follows that for all $t \geq T$, we have

$$
|X(t)| \leq|X|_{[-g, T]} e^{\frac{\ln (\delta)}{T+g}(t-T)}+\frac{1}{1-\delta} \sqrt{\frac{p_{l}}{p_{s}}} \sup _{u \in[0, t-T]} \int_{u}^{u+T} e^{-\frac{1}{2} \int_{\ell}^{u+T} p(a) \mathrm{d} a} \lambda(\ell) \mathrm{d} \ell .
$$

Then our final estimate (23) follows from our choice of $p_{*}>0$.

Remark 1. Although (23) only holds when $t \geq T$, we can use the following Gronwall inequality argument to extend $(23)$ to all of $[0, \infty)$ so that it becomes an ISS estimate. The first inequality in (25) (with the choice $r=0)$ and our lower bound $-p_{*}$ on $p(t)$ imply that for all $t \in[0, T]$, we have

$$
\begin{aligned}
|X(t)| \leq & \sqrt{\frac{p_{l}}{p_{s}}} e^{\frac{T p_{*}}{2}}|X(0)|+\sqrt{\frac{p_{l}}{p_{s}}} \int_{0}^{t} e^{\frac{t-\ell}{2} p_{*}} \mu(\ell) \sup _{m \in[\ell-g, \ell]}|X(m)| \mathrm{d} \ell+\sqrt{\frac{p_{l}}{p_{s}}} \int_{0}^{t} e^{\frac{t-\ell}{2} p_{*}} \lambda(\ell) \mathrm{d} \ell \\
\leq & \left(\sqrt{\frac{p_{l}}{p_{s}}} e^{\frac{T p_{*}}{2}}+\sqrt{\frac{p_{l}}{p_{s}}} \int_{0}^{t} e^{\frac{t-\ell}{2} p_{*}} \mu(\ell) \mathrm{d} \ell\right)|X|_{[-g, 0]}+\sqrt{\frac{p_{l}}{p_{s}}} \int_{0}^{t} e^{\frac{t-\ell}{2} p_{*}} \lambda(\ell) \mathrm{d} \ell \\
& +\sqrt{\frac{p_{l}}{p_{s}}} \int_{0}^{t} e^{\frac{t-\ell}{2} p_{*}} \mu(\ell) \sup _{m \in[0, \ell]}|X(m)| \mathrm{d} \ell \\
\leq & \theta_{1}(T) \int_{0}^{t} \sup _{m \in[0, \ell]}|X(m)| \mathrm{d} \ell+\theta_{2}(t, T),
\end{aligned}
$$

where

$$
\theta_{1}(T)=\sqrt{\frac{p_{l}}{p_{s}}} e^{\frac{T}{2} p_{*}}|\mu|_{[0, T]} \text { and } \theta_{2}(t, T)=\sqrt{\frac{p_{l}}{p_{s}}} e^{\frac{T p_{*}}{2}}\left(1+T|\mu|_{[0, T]}\right)|X|_{[-g, 0]}+\sqrt{\frac{p_{l}}{p_{s}}} e^{\frac{T p_{*}}{2}} T|\lambda|_{[0, t]} .
$$

Hence, the function $\mathcal{G}(\ell)=\sup _{m \in[0, \ell]}|X(m)|$ is such that

$$
|X(t)| \leq \mathcal{G}(t) \leq \theta_{1}(T) \int_{0}^{t} \mathcal{G}(\ell) \mathrm{d} \ell+\theta_{2}(t, T)
$$

holds for all $t \in[0, T]$. It follows from Gronwall's inequality that for all $t \in[0, T]$, we have

$$
\int_{0}^{t} \mathcal{G}(\ell) \mathrm{d} \ell=\int_{0}^{t} \sup _{m \in[0, \ell]}|X(m)| \mathrm{d} \ell \leq T e^{T \theta_{1}(T)} \theta_{2}(t, T) .
$$

Combining (32) with (33), and then using the fact that $\delta \in(0,1)$ and the definition of $\theta_{2}$, we obtain

$$
\begin{aligned}
|X(t)| \leq & \theta_{1}(T) T e^{T \theta_{1}(T)} \theta_{2}(t, T)+\theta_{2}(t, T) \\
\leq & {\left[\theta_{1}(T) T e^{T \theta_{1}(T)}+1\right] \sqrt{\frac{p_{l}}{p_{s}}} e^{T p_{*} / 2}\left(1+T|\mu|_{[0, T]}\right) e^{\frac{\ln (\delta)}{T+g}(t-T)}|X|_{[-g, 0]} } \\
& +\left[\theta_{1}(T) T e^{T \theta_{1}(T)}+1\right] \sqrt{\frac{p_{l}}{p_{s}}} e^{T p_{*} / 2} T|\lambda|_{[0, t]}
\end{aligned}
$$


for all $t \in[0, T]$. Since $\theta_{2}(T, T) \geq|X|_{[-g, 0]}$, it follows from (23) and the first inequality in (34) that

$$
\begin{aligned}
& |X(t)| \leq \max \left\{|X|_{[-g, 0]},\left(\theta_{1}(T) T e^{T \theta_{1}(T)}+1\right) \theta_{2}(T, T)\right\} e^{\frac{\ln (\delta)}{T+g}(t-T)}+\frac{2 \sqrt{p_{l}}\left(e^{T p_{*} / 2}-1\right)}{p_{*}(1-\delta) \sqrt{p_{s}}}|\lambda|_{[0, t]} \\
& \leq\left(\theta_{1}(T) T e^{T \theta_{1}(T)}+1\right) \sqrt{\frac{p_{l}}{p_{s}}} e^{\frac{T p_{*}}{2}}\left(1+T|\mu|_{[0, T]}\right)|X|_{[-g, 0]} e^{\frac{\ln (\delta)}{T+g}(t-T)} \\
& +\left[\left(\theta_{1}(T) T e^{T \theta_{1}(T)}+1\right) \frac{T \sqrt{p_{l}} e^{\frac{T p_{*}}{2}}}{\sqrt{p_{s}}}+\frac{2 \sqrt{p_{l}}\left(e^{T p_{*} / 2}-1\right)}{p_{*}(1-\delta) \sqrt{p_{s}}}\right]|\lambda|_{[0, t]}
\end{aligned}
$$

holds for all $t \geq T$, where the second inequality used the fact that $X(t)$ does not depend on values $\lambda(t)$ for $t \geq T$, so we can replace $\theta_{2}(T, T)$ by $\theta_{2}(t, T)$. Comparing (34)-(35), it follows that (35) holds for all $t \geq 0$.

\section{Delayed Systems with Stabilizing Undelayed Parts}

\section{IV.1 Statement and Proof of Result}

We next consider systems of the form

$$
\dot{x}(t)=\left[A(t)+\delta_{A}(t)\right] x(t)+\left[B(t)+\delta_{B}(t)\right] x(t-h(t))+\varepsilon(t)
$$

whose stabilizing part $A(t) x(t)$ consists of a term without delay; but see the next section for other cases. We assume that the state $x$ is valued in $\mathbb{R}^{n}$, the initial conditions are in $C_{\text {in }}, h:[0, \infty) \rightarrow[0, \infty)$ is a piecewise continuous function, and $A:[0, \infty) \rightarrow \mathbb{R}^{n \times n}, B:[0, \infty) \rightarrow \mathbb{R}^{n \times n}$, and $\varepsilon:[0, \infty) \rightarrow \mathbb{R}^{n}$ are locally bounded piecewise continuous functions. The matrix valued functions $A$ and $B$ are known, but the additive uncertainties $\delta_{A}(t)$ and $\delta_{B}(t)$ are unknown bounded piecewise continuous functions. The functions $\varepsilon$ also represent uncertainty. Although it is possible to remove $\delta_{A}$ and $\delta_{B}$ in (36) and simply assume that the coefficient matrices $A$ and $B$ are uncertain, it is important for what follows to view the vector field $A(t)+\delta_{A}(t)$ in $(36)$ as being a sum of known and unknown matrices as in (36), because of how our assumptions involve $A$.

One consequence of our second theorem will be that even when the term $B(t) x(t-h(t))$ does not help stabilize the system, we can prove key asymptotic stability properties in cases where classical stability analysis techniques (such as Razumikhin's theorem or Lyapunov-Krasovskii functional constructions) do not seem to apply; see Section VIII for examples. We introduce the undelayed system

$$
\dot{z}(t)=A(t) z(t)
$$

with $z$ valued in $\mathbb{R}^{n}$ and the following two assumptions:

Assumption 1. There exist a continuous function $p_{1}:[0, \infty) \rightarrow \mathbb{R}$, a $C^{1}$ function $P:[0, \infty) \rightarrow \mathbb{R}^{n \times n}$ such that $P(t)$ is symmetric and positive definite for all $t \geq 0$, and positive constants $p_{2}, p_{3}$, and $p_{4}$ such that

$$
p_{1}(t) \geq-p_{4} \text { and } p_{2}|z|^{2} \leq V(t, z) \leq p_{3}|z|^{2}
$$

hold for all $t \geq 0$ and $z \in \mathbb{R}^{n}$, where

$$
V(t, z)=z^{\top} P(t) z
$$

and such that along all trajectories $z(t)$ of $(37)$, we have

$$
\frac{d}{d t} V(t, z(t)) \leq-p_{1}(t) V(t, z(t))
$$

for all $t \geq 0$. Also, the function $h(t)$ is locally bounded and piecewise continuous.

Assumption 2. There is a constant $g \geq 0$ such that

$$
0 \leq h(t) \leq g \text { for all } t \geq 0 .
$$

Also, there are constants $T>g$ and $\delta \in(0,1)$ such that

$$
\sqrt{\frac{p_{3}}{p_{2}}}\left[e^{-\frac{1}{2} \int_{t-T}^{t} p_{1}(m) \mathrm{d} m}+\int_{t-T}^{t} e^{-\frac{1}{2} \int_{\ell}^{t} p_{1}(m) \mathrm{d} m}\left(\left|\delta_{A}(\ell)\right|+\left|B(\ell)+\delta_{B}(\ell)\right|\right) \mathrm{d} \ell\right] \leq \delta \text { for all } t \geq T,
$$

where the $p_{i}$ 's are from Assumption 1. 
See Section IV.2 for ways to check the preceding assumptions. Our first theorem for (36) is as follows:

Theorem 2. If Assumptions 1-2 hold, then, with the choices

$$
\begin{aligned}
& \mathcal{H}_{1}=\left(\theta_{1}(T) T e^{T \theta_{1}(T)}+1\right) \sqrt{\frac{p_{3}}{p_{2}}} e^{T p_{4} / 2}, \Delta(t)=\left|\delta_{A}(t)\right|+\left|B(t)+\delta_{B}(t)\right|, \\
& \text { and } \theta_{1}(T)=\sqrt{\frac{p_{3}}{p_{2}}} e^{T p_{4} / 2}|\Delta|_{[0, T]},
\end{aligned}
$$

all solutions $x(t)$ of (36) satisfy

$$
|x(t)| \leq\left(1+T|\Delta|_{[0, T]}\right) \mathcal{H}_{1}|x|_{[-g, 0]} e^{\frac{\ln (\delta)}{T+g}(t-T)}+\left(\mathcal{H}_{1} T+\frac{2}{(1-\delta) p_{4}} \sqrt{\frac{p_{3}}{p_{2}}}\left(e^{T p_{4} / 2}-1\right)\right)|\varepsilon|_{[0, t]}
$$

for all $t \geq 0$.

Proof. Set

$$
\mathcal{R}\left(t, x_{t}\right)=\delta_{A}(t) x(t)+\left[B(t)+\delta_{B}(t)\right] x(t-h(t))+\varepsilon(t) .
$$

Then (36) can be rewritten as $\dot{x}(t)=A(t) x(t)+\mathcal{R}\left(t, x_{t}\right)$. Also, for all $t \geq 0$, we have

$$
\begin{aligned}
\left|\mathcal{R}\left(t, x_{t}\right)\right| & \leq\left|\delta_{A}(t)\right||x(t)|+\left|B(t)+\delta_{B}(t)\right||x(t-h(t))|+|\varepsilon(t)| \\
& \leq\left(\left|\delta_{A}(t)\right|+\left|B(t)+\delta_{B}(t)\right|\right) \sup _{m \in[t-g, t]}|x(m)|+|\varepsilon(t)|
\end{aligned}
$$

We now apply Theorem 1 , with the choices $\mathcal{U}=\mathcal{R}, \mu=\Delta, \mathcal{F}=A, p_{l}=p_{3}, p_{s}=p_{2}, p_{*}=p_{4}$, and $\lambda(t)=|\varepsilon(t)|$. Then (44) follows from (35) in Remark 1.

\section{IV.2 Interesting Features of Theorem 2}

Before turning to our next theorem, we discuss several notable features of Theorem 2. A striking feature of our final ISS estimate (44) is that the overshoot is zero when $\varepsilon=0$, in which case we have exponential stability of the origin even when there are additive uncertainties $\delta_{A}$ and $\delta_{B}$ on $A$ and $B$. Also, we do not require $p_{1}$ to be nonnegative valued. On the other hand, Assumptions 1-2 imply that (37) is globally exponentially stable to zero (GES) when $\delta_{A}=\delta_{B}=0$, since we can take $B=0$ and $\varepsilon=0$. Since we do not require $p_{1}$ to be periodic, it does not seem possible to transform $V$ into a strict Lyapunov function for (37). We can sometimes prove exponential stability by using Razumikhin's theorem, but not always, as we illustrate in our examples below.

Theorem 2 can be used to provide a bound on $B+\delta_{B}$ such that (36) is GES if (37) is GES. Moreover, we can find a bound on $B+\delta_{B}$ that is independent of the bound $g$ on $h(t)$. To check that this can be done, we first assume that (37) is GES and that $A$ is bounded and continuous; see below for more general cases. For simplicity, we assume that the perturbation terms $\varepsilon$ and $\delta_{A}$ in (36) are zero, but analogous reasoning applies when $\varepsilon$ and $\delta_{A}$ are nonzero. Then standard results (such as [25, Theorem 4.14]) make it possible to satisfy Assumption 1 using a constant positive value of $p_{1}(t)=\bar{p}_{1}$. Also, (42) holds if there is a constants $T>0$ such that

$$
e^{-T \bar{p}_{1} / 2}+\left|B+\delta_{B}\right|_{[0, \infty)} \int_{0}^{T} e^{-\bar{p}_{1} r / 2} \mathrm{~d} r<\sqrt{\frac{p_{2}}{p_{3}}},
$$

or, equivalently, if $\left|B+\delta_{B}\right|_{[0, \infty)}<\left(\bar{p}_{1} / 2\right)\left(\sqrt{p_{2} / p_{3}}-e^{-T \bar{p}_{1} / 2}\right)\left(1-e^{-T \bar{p}_{1} / 2}\right)^{-1}$.

Here is an analog where $p_{1}$ can take both positive and negative values. Assume that

$$
\dot{z}(t)=\mathcal{N} z(t)
$$

is GES, where $\mathcal{N} \in \mathbb{R}^{n \times n}$ is constant, and consider any bounded continuous function $q: \mathbb{R} \rightarrow \mathbb{R}$ for which there are constants $q_{0}>0$ and $T>0$ such that

$$
\int_{t-T}^{t} q(\ell) \mathrm{d} \ell \geq q_{0}
$$

for all $t \in \mathbb{R}$. This allows cases where $q$ is a persistency of excitation function such as $q(\ell)=\sin ^{2}(\ell)$ (by choosing $T=\pi$ and $\left.q_{0}=\pi / 2\right)$, or a function such as $q(\ell)=\sin (\pi \ell / T)+3 / \pi$ that takes both positive and negative values (by taking any constant $T>0$ and $q_{0}=T / \pi$ ). By solving a Riccati equation, we can build a symmetric positive 
definite matrix $P$ and also find positive constants $\underline{\alpha}, \bar{\alpha}$, and $\alpha_{*}$ such that the time derivative of $V(z)=z^{\top} P z$ along all trajectories $z(t)$ of (48) satisfies

$$
\frac{d}{d t} V(z(t))=z(t)^{\top}\left(\mathcal{N}^{\top} P+P \mathcal{N}\right) z(t) \leq-\alpha_{*} V(z(t))
$$

and $\underline{\alpha}|z|^{2} \leq V(z) \leq \bar{\alpha}|z|^{2}$ for all $z \in \mathbb{R}^{n}$, e.g., by taking $\underline{\alpha}=\lambda_{\min }(P)$ and $\bar{\alpha}=\lambda_{\max }(P)$. Set $A(t)=q(t) \mathcal{N}$. Then Assumption 1 holds with $p_{1}(t)=\alpha_{*} q(t), p_{2}=\underline{\alpha}, p_{3}=\bar{\alpha}$, and $p_{4}=\left|p_{1}\right|_{[0, \infty)}$. Then for each constant $g>0$, we can argue as in the preceding paragraph to conclude that

$$
\dot{x}(t)=q(t) \mathcal{N} x(t)+\left(B(t)+\delta_{B}(t)\right) x(t-h(t))
$$

is GES for all piecewise continuous functions $h:[0, \infty) \rightarrow[0, g]$, and all choices of $B: \mathbb{R} \rightarrow \mathbb{R}^{n \times n}$ such that

$$
\left|B+\delta_{B}\right|_{[0, \infty)}<\inf _{t \geq T}\left(\int_{t-T}^{t} \exp \left(-\frac{\alpha_{*}}{2} \int_{m}^{t} q(\ell) \mathrm{d} \ell\right) \mathrm{d} m\right)^{-1}\left(\sqrt{\frac{\alpha}{\bar{\alpha}}}-\exp \left(-\frac{q_{0} \alpha_{*}}{2}\right)\right)
$$

holds. In fact, the GES property still holds if we replace (52) by the weaker condition

$$
\sup _{t \geq T} \frac{1}{T} \int_{t-T}^{t} \exp \left(-\frac{\alpha_{*}}{2} \int_{m}^{t} q(\ell) \mathrm{d} \ell\right)\left|B(m)+\delta_{B}(m)\right| \mathrm{d} m<\frac{1}{T}\left(\sqrt{\frac{\alpha}{\bar{\alpha}}}-\exp \left(-\frac{q_{0} \alpha_{*}}{2}\right)\right),
$$

which simply says that $\left|B+\delta_{B}\right|$ is small in a suitable averaged sense. We can use similar reasoning if $\mathcal{N}$ is time-varying, provided (a) $\dot{z}=\mathcal{N}(t) z$ is a uniformly globally exponentially stable system that admits a time invariant Lyapunov function of the form $V(z)=z^{\top} P z$ and (b) $\mathcal{N}$ is bounded and piecewise continuous. Hence, Theorem 2 can be viewed as a robustness result for GES of (37) with respect to the potentially destabilizing terms $\left[B(t)+\delta_{B}(t)\right] x(t-h(t))+\varepsilon(t)$.

\section{Systems Whose Undelayed Part Need Not Be Stabilizing}

\section{V.1 Statement and Proof of Result}

We next study systems (36) in the fundamental case where (37) is not necessarily exponentially stable. Consider

$$
\dot{z}(t)=H(t) z(t)
$$

where $H(t)=A(t)+B(t)$. We make the following assumption:

Assumption 3. The matrices $A, \delta_{A}, B$, and $\delta_{B}$ and $h$ are locally bounded and piecewise continuous, and there is a constant $g$ such that $0 \leq h(t) \leq g$ for all $t \geq 0$. Also, there are a bounded piecewise continuous function $p_{1}:[0, \infty) \rightarrow \mathbb{R}$, positive constants $p_{2}$ and $p_{3}$, and a $C^{1}$ function $P:[0, \infty) \rightarrow \mathbb{R}^{n \times n}$ such that $P(t)$ is symmetric and positive definite for all $t \geq 0$, for which: For all $t \geq 0$ and $z \in \mathbb{R}^{n}$, we have

$$
p_{2}|z|^{2} \leq V(t, z) \leq p_{3}|z|^{2},
$$

where $V(t, z)=z^{\top} P(t) z$ and the time derivative of $V$ along all trajectories $z(t)$ of (54) satisfies

$$
\frac{d}{d t} V(t, z(t)) \leq-p_{1}(t) V(t, z(t))
$$

for all $t \geq 0$.

We also make the convention that $A, \delta_{A}, B$, and $\delta_{B}$ are 0 on $[-g, 0]$, to extend their domains to $[-g, \infty)$. We then add the following assumption:

Assumption 4. There exist two constants $T>g$ and $\delta \in(0,1)$ such that with the choice

$$
\mu(t)=\left|\delta_{A}(t)+\delta_{B}(t)\right|+\left|B(t)+\delta_{B}(t)\right| \int_{t-h(t)}^{t}\left(\left|A(\ell)+\delta_{A}(\ell)\right|+\left|B(\ell)+\delta_{B}(\ell)\right|\right) \mathrm{d} \ell,
$$

we have

$$
\sqrt{\frac{p_{3}}{p_{2}}}\left(e^{-\frac{1}{2} \int_{t-T}^{t} p_{1}(m) \mathrm{d} m}+\int_{t-T}^{t} e^{-\frac{1}{2} \int_{\ell}^{t} p_{1}(m) \mathrm{d} m} \mu(\ell) \mathrm{d} \ell\right) \leq \delta
$$

for all $t \geq T$, where $g$ is from Assumption 3. Also, $\varepsilon$ is locally bounded and piecewise continuous. 
See Remark 5 for ways to check Assumption 4. The following ISS result reduces to global exponential stability when the perturbations $\varepsilon$ are zero (but also see Remark 2 for results for more general initial functions):

Theorem 3. If the system (36) satisfies Assumptions 3-4, then we can construct a constant $\bar{c}>0$ such that

$$
|x(t)| \leq \bar{c} e^{\frac{\ln (\delta) t}{T+g}}+\bar{c}\left(1+|h|_{[0, t]}\left|B+\delta_{B}\right|_{[0, t]}\right)|\varepsilon|_{[0, t]}
$$

holds along all trajectories of (36) for all $t \geq 0$ and all constant initial functions.

Proof. For all $t \geq 0$, we can rewrite (36) as

$$
\dot{x}(t)=H(t) x(t)+\left\{\left(\delta_{A}(t)+\delta_{B}(t)\right) x(t)-\left(B(t)+\delta_{B}(t)\right)[x(t)-x(t-h(t))]+\varepsilon(t)\right\} .
$$

We apply Theorem 1 . For all $t \geq 0$, we can use the constantness of the initial functions to get

$$
|x(t)-x(t-h(t))| \leq \int_{t-h(t)}^{t}\left(\left|A(\ell)+\delta_{A}(\ell)\right|+\left|B(\ell)+\delta_{B}(\ell)\right|\right) \mathrm{d} \ell|x|_{[t-g, t]}+h(t)|\varepsilon|_{[\max \{0, t-g\}, t]} .
$$

Let $\mathcal{U}\left(t, X_{t}\right)$ denote the quantity in curly braces in (60) for all $t \geq 0$. Then for all $t \geq 0$, we get

$$
\dot{x}(t)=H(t) x(t)+\mathcal{U}\left(t, x_{t}\right),
$$

where

$$
|\mathcal{U}(t, \phi)| \leq \mu(t)|\phi|_{[-g, 0]}+\lambda(t)
$$

for all $t \geq 0$ and all $\phi \in C\left([-g, 0], \mathbb{R}^{n}\right)$, and where $\mu$ is from (57), and

$$
\lambda(t)=\left.\left(1+h(t)\left|B+\delta_{B}\right|_{[0, t]}\right)\right|_{\left.\varepsilon\right|_{[0, t]}} .
$$

Then all assumptions from Theorem 1 hold, with $X=x, p(t)=p_{1}(t), p_{l}=p_{3}$, and $p_{s}=p_{2}$, so the theorem follows from Theorem 1 and our ISS estimate (35) from Remark 1.

Remark 2. The upper bound in (61) was used to obtain the bound (63) which was in turn used to apply the stability condition from Theorem 1. In some cases, the upper bound in (61) may be significantly larger than the left side of (61), which may introduce conservativeness in our stability conditions. However, Theorem 3 presents the advantage of being based on easily checkable conditions, while the determination of the necessary and sufficient conditions for stability for (36) is an open problem, which seems to be formidably difficult.

Although we used the constantness of the initial functions to prove (61), we can generalize the preceding theorem to cover nonconstant initial functions, as follows: If $t \geq 0$ is such that $t \geq h(t)$, then (61) still holds, even if the initial functions are not constant. On the other hand, if $t \geq 0 \geq t-h(t)$, then

$$
|x(t)-x(t-h(t))| \leq|x(t)-x(0)|+|x(0)-x(t-h(t))| \leq|x(t)-x(0)|+h(t)|\dot{x}|_{[-g, 0]},
$$

and we can upper bound $|x(t)-x(0)|$ by the right side of $(61)$. Therefore, we can argue as in the last part of the proof of Theorem 3 to obtain

$$
|x(t)| \leq \bar{c} e^{\frac{\ln (\delta) t}{T+g}}+\bar{c}\left(1+|h|_{[0, t]}\left|B+\delta_{B}\right|_{[0, t]}\right)|\varepsilon|_{[0, t]}+\bar{c}|h|_{[0, t]}|\dot{x}|_{[-g, 0]}
$$

along all trajectories of (36) for all $t \geq 0$ and all essentially bounded absolutely continuous initial functions.

\section{V.2 Notable Features of Theorem 3}

Remark 3. Our assumptions on $A, \delta_{A}, B$, and $\delta_{B}$ ensure that all solutions of (36) are well-defined and defined over $[0, \infty)$. As before, we do not require $p_{1}$ to be nonnegative valued. Also, Assumptions 3-4 imply that the system (54) is globally exponentially stable to 0 (by taking $\delta_{A}, \delta_{B}, h$, and $\varepsilon$ to be zero), and we can use (35) as before to construct explicit comparison functions $\beta \in \mathcal{K} \mathcal{L}$ and $\gamma \in \mathcal{K}_{\infty}$ in the final ISS estimate.

Remark 4. Theorem 3 is beyond the scope of [17, Chapt. 6], and can be extended to systems with different time-varying delays in different components of the state. Through Assumption 3 in the special case where $\delta_{B}=0$, Theorem 3 takes into account the case where the term $B(t) x(t-h(t))$ has a stabilizing effect. 
Remark 5. To motivate Assumption 4, let us consider the special case where the initial functions, $p_{1}, P, A$ and $B$ are all constant, and where the uncertainties $\delta_{A}$ and $\delta_{B}$ are zero. Then (58) holds if

$$
\sqrt{\frac{p_{3}}{p_{2}}}\left(e^{-T p_{1} / 2}+|B|(|A|+|B|) \int_{t-T}^{t} h(\ell) e^{(\ell-t) p_{1} / 2} \mathrm{~d} \ell\right) \leq \delta .
$$

When $B \neq 0$, the preceding inequality holds provided there are constants $T>0$ and $\delta \in(0,1)$ such that

$$
\frac{1}{T} \int_{t-T}^{t} h(m) e^{(m ;-t) p_{1} / 2} \mathrm{~d} m \leq \frac{\delta \sqrt{p_{2} / p_{3}}-e^{-T p_{1} / 2}}{T|B|(|A|+|B|)}
$$

is satisfied for all $t \geq T$, which is related to an average value of $h$.

\section{Systems with Reduction Controls and Uncertain Delays}

Although there did not exist any explicit controls in Theorems 2-3, they can be viewed as results on timevarying linear systems with time-varying linear state feedbacks with input delays, such as those that arise from tracking problems with latencies. On the other hand, we noted in the introduction that dynamic controllers are an important alternative to state feedbacks that have the potential advantage that they can often compensate for arbitrarily long input delays. Therefore, it is important to develop analogs of Theorems 2-3 for dynamic controllers. In the next two sections, we provide two such analogs. This section provides an analog for reduction method controllers, and the next section covers a different class of dynamic controllers with several useful degrees of freedom.

To highlight the most significant ideas, we confine our analysis in this section to systems of the form

$$
\dot{x}(t)=\left[A+\delta_{A}(t)\right] x(t)+\left[B+\delta_{B}(t)\right] u(t-h(t)-\gamma(t))
$$

where $A \in \mathbb{R}^{n \times n}$ and $B \in \mathbb{R}^{n \times m}$ are known constant matrices, but analogous results can be shown for cases where $A$ and $B$ are time-varying, or where there is additive uncertainty on the right side. Here, $u$ is the control, $h$ is a delay, and $\delta_{A}, \delta_{B}$, and $\gamma$ represent uncertainty. Our first assumption is:

Assumption 5. The function $h$ is of class $C^{1}$ and there is a constant $g>0$ such that $0 \leq h(t) \leq g$ holds for all $t \geq 0$. The unknown function $\gamma$ is piecewise continuous, and there is a known constant $\gamma_{c} \geq 0$ such that the inequalities $0 \leq \gamma(t) \leq \gamma_{c}$ hold for all $t \geq 0$.

Assumption 6. The pair $(A, B)$ is controllable, and $\delta_{A}$ and $\delta_{B}$ are locally bounded and piecewise continuous.

Assumption 6 provides a matrix $K \in \mathbb{R}^{m \times n}$ such that $R=A+B K$ is Hurwitz. Hence, we can find a constant $c>0$ and a positive definite symmetric matrix $Q \in \mathbb{R}^{n \times n}$ for which

$$
Q R+R^{\top} Q \leq-c Q
$$

We also introduce the functions

$$
\begin{aligned}
& \mu_{a}(t)=e^{|A| h(t)}\left(\left(e^{|A| h(t)}+|B K| \mathcal{L}(|A|, h(t))\right)\left|\delta_{A}\right|_{[0, t]}+\left|\delta_{B}\right|_{[0, t]}|K|\right) \text { and } \\
& \mu_{b}(t)=|\dot{h}(t)|\left(|A|+\left(2 e^{|A| h(t)}-1\right)|B K|\right)+2 e^{|A| h(t)}|B K|
\end{aligned}
$$

where $\mathcal{L}(|A|, h(t))=\left(e^{|A| h(t)}-1\right) /|A|$ when $|A| \neq 0$ and $\mathcal{L}(0, h(t))=h(t)$, so $\mu_{a}$ is zero when $\delta_{A}$ and $\delta_{B}$ are zero. Finally, we introduce this assumption:

Assumption 7. There exists a constant $\delta \in(0,1)$ such that

$$
\sqrt{\frac{\lambda_{\max }(Q)}{\lambda_{\min }(Q)}}\left[e^{-c\left(\gamma_{c}+g\right) / 2}+\int_{t-\gamma_{c}-g}^{t} e^{-c(t-\ell) / 2}\left(\mu_{a}(\ell)+\mu_{b}(\ell)\right) \mathrm{d} \ell\right] \leq \delta
$$

holds for all $t \geq \gamma_{c}+g$. 
We prove the following result:

Theorem 4. If Assumptions 5-7 hold, then for any initial function for $u$, the control law

$$
u(t)=K\left[e^{A h(t)} x(t)+\int_{t-h(t)}^{t} e^{A(t-\ell)} B u(\ell) \mathrm{d} \ell\right]
$$

renders the origin of (69) globally exponentially stable to 0.

Proof. In what follows, all inequalities and equalities should be understood to hold for all $t \geq 0$, unless otherwise indicated. Set $q(t)=|\dot{h}(t)|^{2}$. Let us introduce the operator

$$
\zeta(t)=e^{A h(t)} x(t)+\Gamma(t), \text { where } \Gamma(t)=\int_{t-h(t)}^{t} e^{A(t-\ell)} B u(\ell) \mathrm{d} \ell .
$$

Then the control $(73)$ is $u(t)=K \zeta(t)$. Also, $\dot{\Gamma}(t)=A \Gamma(t)+B u(t)-(1-\dot{h}(t)) e^{A h(t)} B u(t-h(t))$. Since the system (69) can be written as

$$
\dot{x}(t)=A x(t)+B u(t-h(t)-\gamma(t))+\delta_{A}(t) x(t)+\delta_{B}(t) u(t-h(t)-\gamma(t)),
$$

the time derivative of $\zeta$ along all trajectories of $(69)$ is

$$
\begin{aligned}
\dot{\zeta}(t)= & e^{A h(t)} \dot{x}(t)+\dot{h}(t) A e^{A h(t)} x(t)+A \Gamma(t)+B u(t)-(1-\dot{h}(t)) e^{A h(t)} B u(t-h(t)) \\
= & {[1+\dot{h}(t)] A \zeta(t)+B u(t)-\dot{h}(t) A \int_{t-h(t)}^{t} e^{A(t-\ell)} B u(\ell) \mathrm{d} \ell+\dot{h}(t) e^{A h(t)} B u(t-h(t)) } \\
& +e^{A h(t)} B[u(t-h(t)-\gamma(t))-u(t-h(t))]+e^{A h(t)}\left(\delta_{A}(t) x(t)+\delta_{B}(t) u(t-h(t)-\gamma(t))\right),
\end{aligned}
$$

where the second equality used the relation $e^{A h(t)} A x(t)+A \Gamma(t)=A \zeta(t)$ twice. Then the fact that our control (73) is $u(t)=K \zeta(t)$, combined with our choice $R=A+B K$, give

$$
\dot{\zeta}(t)=R \zeta(t)+\mathcal{U}\left(t, \zeta_{t}\right)
$$

for all $t \geq 0$, where

$$
\begin{aligned}
\mathcal{U}\left(t, \zeta_{t}\right) & =\dot{h}(t) A \zeta(t)+\omega\left(t, \zeta_{t}\right)+\kappa\left(t, \zeta_{t}\right)+e^{A h(t)}\left(\delta_{A}(t) x(t)+\delta_{B}(t) K \zeta(t-h(t)-\gamma(t))\right), \\
& \omega\left(t, \zeta_{t}\right)=\dot{h}(t)\left(e^{A h(t)} B K \zeta(t-h(t))-A \int_{t-h(t)}^{t} e^{A(t-\ell)} B K \zeta(\ell) \mathrm{d} \ell\right) \text { and } \\
& \kappa\left(t, \zeta_{t}\right)=e^{A h(t)} B K[\zeta(t-h(t)-\gamma(t))-\zeta(t-h(t))] .
\end{aligned}
$$

We now use (77) to apply Theorem 1 . Notice that

$$
\begin{aligned}
\left|\omega\left(t, \zeta_{t}\right)\right| & \leq|\dot{h}(t)|\left(e^{A \mid h(t)}+|A| \int_{t-h(t)}^{t} e^{|A|(t-\ell)} \mathrm{d} \ell\right)|B K||\zeta|_{[t-g, t]} \\
& =|\dot{h}(t)||B K|\left(2 e^{|A| h(t)}-1\right)|\zeta|_{[t-g, t]} \text { and } \\
\left|\kappa\left(t, \zeta_{t}\right)\right| & \leq 2 e^{|A| h(t)}|B K||\zeta|_{\left[t-g-\gamma_{c}, t\right]}
\end{aligned}
$$

hold for all $t \geq 0$. Also,

$$
\begin{aligned}
|x(t)| & =\left|e^{-A h(t)}(\zeta(t)-\Gamma(t))\right| \leq e^{h(t)|A|}|\zeta(t)|+|B K| \int_{t-h(t)}^{t}\left|e^{A(t-\ell-h(t))}\right| \mathrm{d} \ell|\zeta|_{[t-g, t]} \\
& \leq\left(e^{h(t)|A|}+|B K| \int_{t-h(t)}^{t} e^{|A|(h(t)+\ell-t)} \mathrm{d} \ell\right)|\zeta|_{[t-g, t]} \\
& \leq\left(e^{h(t)|A|}+|B K| \mathcal{L}(|A|, h(t))\right)|\zeta|_{[t-g, t]}
\end{aligned}
$$

for all $t \geq 0$. This leads to

$$
\begin{aligned}
|\mathcal{U}(t, \phi)| \leq & \left\{|\dot{h}(t)|\left(|A|+\left(2 e^{|A| h(t)}-1\right)|B K|\right)+2 e^{|A| h(t)}|B K|\right. \\
& \left.+e^{|A| h(t)}\left(\left(e^{|A| h(t)}+|B K| \mathcal{L}(|A|, h(t))\right)\left|\delta_{A}\right|_{[0, t]}+\left|\delta_{B}\right|_{[0, t]}|K|\right)\right\}|\phi|_{\left[-g-\gamma_{c}, 0\right]}
\end{aligned}
$$

for all $t \geq 0$ and $\phi \in C_{\text {in }}$. Next set $V(\zeta)=\zeta^{\top} Q \zeta$. Then the requirements from (21) hold with $\mathcal{F}=R, p(t)=c$, $p_{s}=\lambda_{\min }(Q)$, and $p_{l}=\lambda_{\max }(Q)$, and (22) agrees with (72) with $T=\gamma_{c}+g$. Hence, the result follows from Theorem 1, combined with the final stability estimate from (35) in Remark 1. 


\section{Perturbed Systems with Different Dynamic Controller}

\section{VII.1 Statement and Proof of Theorem}

We next provide an alternative to Theorem 4 based on a different control law. We study systems of the form

$$
\dot{x}(t)=\left[A+\delta_{A}(t)\right] x(t)+\left[B+\delta_{B}(t)\right] u(t-h(t))+\varepsilon(t),
$$

whose state space is $\mathbb{R}^{n}$, where $A \in \mathbb{R}^{n \times n} \backslash\{0\}$ and $B \in \mathbb{R}^{n \times m}$ are known constant matrices, the unknown locally bounded piecewise continuous functions $\delta_{A}$ and $\delta_{B}$ represent uncertainties, $\varepsilon$ is an unknown locally bounded piecewise continuous disturbance, and the delay $h(t)$ is known, but extensions that allow discontinuous or unknown delays are possible using ideas from the preceding section.

We introduce the following assumption:

Assumption 8. The pair $(A, B)$ is controllable, and there is a constant $g>0$ such that $0 \leq h(t) \leq g$ holds for all $t \geq 0$. Also, $h$ is in $C^{1}$.

Assumption 8 implies that we can construct matrices $A_{f} \in \mathbb{R}^{m \times m}$ and $B_{f} \in \mathbb{R}^{m \times n}$ such that

$$
H=\left[\begin{array}{cc}
A & B \\
B_{f} & A_{f}
\end{array}\right] \in \mathbb{R}^{(n+m) \times(n+m)}
$$

is Hurwitz. See Section VII.2 for ways to construct suitable pairs $\left(A_{f}, B_{f}\right)$. Therefore, we can find a symmetric and positive definite matrix $Q \in \mathbb{R}^{(n+m) \times(n+m)}$ and a constant $c>0$ such that $Q H+H^{\top} Q \leq-c Q$ holds, e.g., by solving the Riccati equation $Q H+H^{\top} Q=-I_{m+n}$ and setting $c=1 / \lambda_{\max }(Q)$. We now set $V(\zeta)=\zeta \zeta^{\top} Q \zeta$ where $\zeta=(\rho, \beta) \in \mathbb{R}^{n} \times \mathbb{R}^{m}$, and we redefine the function $\mu$ by

$$
\mu(\ell)=|\dot{h}(\ell)|\left(|A|+\left(2 e^{h(\ell)|A|}-1\right)|B|\right)+e^{h(\ell)|A|}\left(\left|\delta_{A}(\ell)\right|\left(1+\left(e^{h(\ell)|A|}-1\right) \frac{|B|}{|A|}\right) e^{g|A|}+\left|\delta_{B}(\ell)\right|\right)
$$

which reduces to the much simpler formula $\mu(\ell)=|\dot{h}(\ell)|\left(|A|+\left(2 e^{h(\ell)|A|}-1\right)|B|\right)$ in the important special case where the perturbations $\delta_{A}$ and $\delta_{B}$ are zero. Our final assumption is:

Assumption 9. There exist constants $T>g$ and $\delta \in(0,1)$ such that the inequality

$$
\sqrt{\frac{\lambda_{\max }(Q)}{\lambda_{\min }(Q)}}\left[e^{-c T / 2}+\int_{t-T}^{t} e^{-c(t-\ell) / 2} \mu(\ell) \mathrm{d} \ell\right] \leq \delta
$$

holds for all $t \geq T$.

We can then prove:

Theorem 5. If Assumptions 8-9 hold, then (83) in closed-loop with $u(t)=\beta(t)$, where $\beta$ is any solution of

$$
\dot{\beta}(t)=A_{f} \beta(t)+B_{f}\left[e^{A h(t)} x(t)+\int_{t-h(t)}^{t} e^{A(t-\ell)} B \beta(\ell) \mathrm{d} \ell\right],
$$

is ISS with respect to the set of all locally bounded piecewise continuous functions $\varepsilon:[0, \infty) \rightarrow \mathbb{R}^{n}$.

Proof. Fix any choices of $\varepsilon:[0, \infty) \rightarrow \mathbb{R}^{n}, \delta_{A}:[0, \infty) \rightarrow \mathbb{R}^{n \times n}$, and $\delta_{B}:[0, \infty) \rightarrow \mathbb{R}^{n \times m}$ and let Assumptions 8-9 hold. We define $\rho(t)$ to be the quantity in squared brackets on the right side of $(87)$. Then, $\dot{\beta}(t)=$ $A_{f} \beta(t)+B_{f} \rho(t)$ holds for all $t$. Reorganizing terms gives the following:

$$
\begin{aligned}
\dot{\rho}(t)= & A \dot{h}(t) e^{A h(t)} x(t)+e^{A h(t)}\left(A+\delta_{A}(t)\right) x(t)+e^{A h(t)}\left(B+\delta_{B}(t)\right) u(t-h(t))+e^{A h(t)} \varepsilon(t) \\
& +A\left(\rho(t)-e^{A h(t)} x(t)\right)+B \beta(t)-e^{A h(t)} B \beta(t-h(t))(1-\dot{h}(t)) \\
= & A \rho(t)+e^{A h(t)} \varepsilon(t)+\dot{h}(t) A e^{A h(t)} x(t)+B \beta(t)+\dot{h}(t) e^{A h(t)} B \beta(t-h(t))+\mathcal{R}(t),
\end{aligned}
$$

where $\mathcal{R}(t)=e^{h(t) A}\left[\delta_{A}(t) x(t)+\delta_{B}(t) \beta(t-h(t))\right]$. 
Since $\zeta(t)=(\rho(t), \beta(t)) \in \mathbb{R}^{n+m}$ for all $t \geq 0$, we get

$$
\dot{\zeta}(t)=H \zeta(t)+\left\{\dot{h}(t)\left[\begin{array}{c}
\psi(t) \\
0
\end{array}\right]+\left[\begin{array}{c}
e^{A h(t)} \varepsilon(t)+\mathcal{R}(t) \\
0
\end{array}\right]\right\}
$$

where $\psi(t)=A e^{A h(t)} x(t)+e^{A h(t)} B \beta(t-h(t))$. We next apply Theorem 1 with the choices $X=(\rho, \beta)$, the constant matrix $\mathcal{P}(t)=Q$, the constant function $p(t)=c, p_{s}=\lambda_{\min }(Q), p_{l}=\lambda_{\max }(Q)$, and $\mathcal{U}\left(t, X_{t}\right)$ being the quantity in curly braces in (89). Since

$$
e^{A h(t)} x(t)=\rho(t)-\int_{t-h(t)}^{t} e^{A(t-\ell)} B \beta(\ell) \mathrm{d} \ell
$$

holds for all $t \geq 0$, we can argue as in (81) to get

$$
\left|e^{A h(t)} x(t)\right| \leq|\rho(t)|+\frac{|B|}{|A|}\left(e^{|A| h(t)}-1\right)|\beta|_{[t-g, t]} \text { and }|x(t)| \leq e^{g|A|}\left(|\rho(t)|+\frac{|B|}{|A|}\left(e^{|A| h(t)}-1\right)|\beta|_{[t-g, t]}\right)
$$

for all $t \geq 0$. This gives

$$
\begin{aligned}
& |\psi(t)| \leq\left(|A|+\left(2 e^{|A| h(t)}-1\right)|B|\right)|\zeta|_{[t-g, t]} \text { and } \\
& |\mathcal{R}(t)| \leq e^{|A| h(t)}\left[\left|\delta_{A}(t)\right|\left(1+\left(e^{|A| h(t)}-1\right)|B| /|A|\right) e^{g|A|}+\left|\delta_{B}(t)\right|\right]|\zeta|_{[t-g, t]},
\end{aligned}
$$

and therefore also $|\mathcal{U}(t, \phi)| \leq \mu(t)|\phi|_{[-g, 0]}+\lambda(t)$ for all $t \geq 0$ and $\phi \in C_{\text {in }}$, where $\lambda(t)=e^{g|A|}|\epsilon(t)|$ for all $t \geq 0$ and $\mu$ is defined in (85). The theorem now follows from Theorem 1 , since the contractiveness requirement (22) from Theorem 1 follows from our condition (86).

Remark 6. A useful feature of the preceding reasoning is that it can give explicit formulae for the comparison functions $\beta \in \mathcal{K} \mathcal{L}$ and $\gamma \in \mathcal{K}_{\infty}$ in the final ISS estimate. This follows from Remark 1.

\section{VII.2 Coefficient Matrices in Dynamic Extension}

Theorem 5 requires a pair $\left(A_{f}, B_{f}\right) \in \mathbb{R}^{m \times m} \times \mathbb{R}^{m \times n}$, a positive definite symmetric matrix $Q$, and a constant $c>0$ such that (84) is Hurwitz and such that $Q H+H^{\top} Q \leq-c Q$. Different choices of $A_{f}, B_{f}$, and $Q$ lead to different controllers $u$ in Theorem 5 and different conditions in Assumption 9, so it is of interest to find general ways to build $A_{f}, B_{f}$, and $Q$. (See [43] for a possible choice of the pair $\left(A_{f}, B_{f}\right)$.) On the other hand, [36] builds a large class of pairs $\left(A_{f}, B_{f}\right)$ that satisfy the preceding requirements, and that also contain a useful scaling tuning parameter. For completeness, we state our construction from [36] in the sequel.

Proposition 1. Let $(A, B) \in \mathbb{R}^{n \times n} \times \mathbb{R}^{n \times m}$ be any controllable pair, and $K \in \mathbb{R}^{m \times n}$ be such that $M=A+B K$ is Hurwitz. Let $P \in \mathbb{R}^{n \times n}$ be a positive definite symmetric matrix and $c>0$ be a constant such that

$$
P M+M^{\top} P \leq-c P
$$

and let $\nu>0$ be any constant. Then (a) the matrices $A_{f}=K B-\frac{c}{2} I_{m}$ and $B_{f}=K A-\frac{2}{\nu} B^{\top} P+\frac{c}{2} K$ are such that (84) is Hurwitz and (b) the matrix $V(\zeta)=\zeta^{\top} Q \zeta$ with the choice

$$
Q=\left[\begin{array}{cc}
P+\frac{\nu}{2} K^{\top} K & -\frac{\nu}{2} K^{\top} \\
-\frac{\nu}{2} K & \frac{\nu}{2} I_{m}
\end{array}\right]
$$

is such that $\dot{V}(t) \leq-c V(\zeta)$ holds along all trajectories of $\dot{\zeta}=H \zeta$.

Proof. We have $V(\zeta)=\rho^{\top} P \rho+0.5 \nu(\beta-K \rho)^{\top}(\beta-K \rho)$ for all $\zeta=(\rho, \beta) \in \mathbb{R}^{n} \times \mathbb{R}^{m}$. Hence, by using the Riccati inequality $(92)$, it follows that its time derivative along all trajectories of $\dot{\zeta}=H \zeta$ satisfies

$$
\begin{aligned}
\dot{V}(t) & =2 \rho^{\top} P[M \rho+B(\beta-K \rho)]+\nu(\beta-K \rho)^{\top}\left[A_{f} \beta+B_{f} \rho-K(A \rho+B \beta)\right] \\
& \leq-c \rho^{\top} P \rho+(\beta-K \rho)^{\top}\left[\nu\left(A_{f}-K B\right) \beta+\left(\nu B_{f}+2 B^{\top} P-\nu K A\right) \rho\right] .
\end{aligned}
$$


We choose

$$
A_{f}=K B-\frac{1}{\nu} G_{f} \text { and } B_{f}=\frac{G_{f} K}{\nu}-\frac{2}{\nu} B^{\top} P+K A,
$$

where $G_{f} \in \mathbb{R}^{m \times m}$ is to be chosen. Then

$$
\dot{V}(t) \leq-c \rho^{\top} P \rho+(\beta-K \rho)^{\top}\left[-G_{f} \beta+G_{f} K \rho\right]=-c\left[\rho^{\top} P \rho+\frac{1}{c}(\beta-K \rho)^{\top} G_{f}(\beta-K \rho)\right] .
$$

The proposition now follows by choosing $G_{f}=\frac{\nu}{2} c I_{m}$.

\section{Further Illustrative Examples}

In Section IV.2, we saw how to apply some of our work under persistency of excitation conditions. In this section, we provide additional examples that illustrate the potential of our work.

\section{VIII.1 Illustration of Theorem 2}

Let $d>0$ and $c \in(0,1)$ be any constants such that:

$$
(1-c) d<1-e^{-1 / 2} .
$$

Let $\sigma:[0, \infty) \rightarrow\{0, d\}$ be the periodic function of period 1 defined by (i) $\sigma(t)=0$ when $t \in[0, c)$ and (ii) $\sigma(t)=d$ when $t \in[c, 1]$. We consider the system

$$
\dot{x}(t)=-\left[1+\delta_{A}(t)\right] x(t)+\left[\sigma(t)+\delta_{B}(t)\right] x(t-1),
$$

where $x$ is valued in $\mathbb{R}$, and where the piecewise continuous locally bounded functions $\delta_{A}$ and $\delta_{B}$ represent uncertainties as before. When $d>1$, Razumikhin's theorem does not apply; see [17, Chapt. 6]. To the best of the authors' knowledge, no Lyapunov-Krasovskii functional construction technique applies to this system.

Let us verify that (97) satisfies Assumptions 1-2 with $V(x)=\frac{1}{2} x^{2}, \varepsilon=0$, and $h(t)=1$, under suitable bounds on $\delta_{A}$ and $\delta_{B}$. With the notation from Assumptions 1-2, we choose $p_{1}(t)=1$ and $P(t)=1 / 2$ for all

$t \geq 0$ and the constants $p_{2}=p_{3}=\frac{1}{2}$ and $T=p_{4}=1$. Then Assumption 1 is satisfied with $A=-1$. Also, the requirement (42) from Assumption 2 is that there is a constant $\delta \in(0,1)$ such that

$$
e^{-1 / 2}+\sup _{t \geq 1} \int_{t-1}^{t} e^{-(t-\ell) / 2}\left(\left|\delta_{A}(\ell)\right|+\left|\sigma(\ell)+\delta_{B}(\ell)\right|\right) \mathrm{d} \ell \leq \delta
$$

holds. Since $\int_{t-1}^{t} \sigma(\ell) \mathrm{d} \ell=d(1-c)$ for all $t \geq 1$, it follows that (98) holds for some constant $\delta \in(0,1)$ if

$$
\sup _{t \geq 1} \int_{t-1}^{t} e^{-(t-\ell) / 2}\left(\left|\delta_{A}(\ell)\right|+\left|\delta_{B}(\ell)\right|\right) \mathrm{d} \ell<1-e^{-1 / 2}-d(1-c),
$$

and the right side of (99) is positive, by (96). Theorem 2 allows us to conclude that the corresponding perturbed systems are globally exponentially stable to 0, when (99) holds. More generally, we can use Theorem 2 to cover cases where the system has time-varying delays, or additive uncertainty $\varepsilon(t)$ on the right side of (97) to conclude input-to-state stability. We leave these generalizations to the reader.

\section{VIII.2 Illustration of Theorem 3}

Let $l>0$ be a constant, and $k \in \mathbb{N}$ be an odd integer. We consider the system

$$
\dot{x}(t)=q(t) x(t)-\left(1-\delta_{B}(t)\right) x\left(t-h_{k}(t)\right),
$$

where $x$ is valued in $\mathbb{R}$, the bounded piecewise continuous nonnegative valued function $q$ admits a constant $T \in(0,1)$ such that the constant $\bar{q}$ defined by

$$
\bar{q}=\sup _{t \geq T} \int_{t-T}^{t} q(\ell) \mathrm{d} \ell
$$


satisfies $\bar{q}<T$, and the time-varying delay is $h_{k}(t)=\max \left\{0, l \sin ^{k}(t)\right\}$. Notice that this allows cases where $q(\ell)>1$ for some choices of $\ell$, as long the averaged value $\bar{q} / T$ is below 1 . To the best of the authors' knowledge, Razumikhin's theorem does not apply to (100), and no Lyapunov-Krasovskii functional construction technique in the literature applies to this system.

With the notation of Section V.1, we choose $P(t)=1, p_{1}(t)=2(1-q(t)), p_{2}=p_{3}=1, H(t)=q(t)-1$, $A(t)=q(t)$, and $B=-1$. Even though $p_{1}(t)$ can take both positive and negative values, Assumption 3 is still satisfied. Also, the inequality (58) from Assumption 4 holds if there is a constant $\delta \in(0,1)$ such that

$$
\begin{aligned}
& e^{-T+\bar{q}}+\int_{t-T}^{t} \exp \left(r-t+\int_{r}^{t} q(a) \mathrm{d} a\right) \mu(r) \mathrm{d} r \leq \delta \text { for all } t \geq T, \\
& \text { where } \mu(r)=\left|\delta_{B}(r)\right|+\left|\delta_{B}(r)-1\right| \int_{r-h_{k}(r)}^{r}\left(|q(\ell)|+\left|\delta_{B}(\ell)-1\right|\right) \mathrm{d} \ell .
\end{aligned}
$$

Pick any constant $\bar{\delta}>0$ such that $e^{-T+\bar{q}}+T e^{\bar{q}} \bar{\delta}<1$. We now allow only those piecewise continuous perturbations $\delta_{B}$ such that $\left|\delta_{B}\right|_{[0, \infty)} \leq \bar{\delta}$. By the Lebesgue Dominated Convergence Theorem, there is a constant $k>0$ (which is sufficiently large, and which depends on $l, T,|q|_{[0, \infty)}$, and $\bar{\delta}$ ) such that there exists some constant $\delta \in(0,1)$ satisfying $(102)$ for all $t \geq T$, by the following argument. First, notice that the inequality from (102) holds if

$$
\int_{t-T}^{t} \exp \left(r-t+\int_{r}^{t} q(a) \mathrm{d} a\right) h_{k}(r) \mathrm{d} r<\frac{\delta-e^{-T+\bar{q}}-T e^{\bar{q}} \bar{\delta}}{\delta+\left(|q|_{[0, \infty)}+\left|\delta_{B}-1\right|_{[0, \infty)}\right)\left|\delta_{B}-1\right|_{[0, \infty)}},
$$

where the $\delta$ in the denominator on the right side of (103) is to allow the case where $\left(|q|_{[0, \infty)}+\left|\delta_{B}-1\right|_{[0, \infty)}\right) \mid \delta_{B}-$ $\left.1\right|_{[0, \infty)}=0$. Also, for each constant $t \geq T$, we can use the Dominated Convergence Theorem to get

$$
\lim _{k \rightarrow \infty} \int_{t-T}^{t} \exp \left(r-t+\int_{r}^{t} q(a) \mathrm{d} a\right) h_{k}(r) \mathrm{d} r=0 .
$$

There is an odd integer $k>0$ such that (103) holds for all $t \geq T$ with $\delta=0.5\left(1+e^{-T+\bar{q}}+T e^{\bar{q}} \bar{\delta}\right)$, provided the convergence in (104) is uniform in $t \geq T$. The convergence (104) will be uniform in $t \geq T$ if the convergence

$$
\lim _{k \rightarrow \infty} \int_{0}^{T} h_{k}(\ell+b) \mathrm{d} \ell=0
$$

is uniform in $b \in[0,2 \pi]$, by the periodicity of the sin function. On the other hand, the functions $f_{k}(b)=$ $\int_{0}^{T} h_{k}(\ell+b) \mathrm{d} \ell$ are continuous in $b$ and they satisfy $\lim _{k \rightarrow \infty} f_{k}(b)=0$ for all $b \in[0,2 \pi]$ (again by the Dominated Convergence Theorem), and $f_{k}(b) \geq f_{k+1}(b)$ for all $k \geq 1$ and $b \in[0,2 \pi]$. Hence, the uniformity of the convergence (104) in $t \geq T$ follows from standard results, e.g., [46, Theorem 7.13, p.150]. Thus, Assumptions 3-4 hold.

Theorem 3 now implies that (100) is globally exponentially stable to 0 for all perturbations $\delta_{B}$ that are bounded by $\bar{\delta}$ and all constant initial functions. More generally, we have the estimate (59) from Theorem 3 , which also allows additive uncertainties $\varepsilon(t)$ on the right side. Since $l>0$ is arbitrarily large, we have exponential stability without any constraint on $\sup _{m \geq 0} h_{k}(m)$. To the best of the authors' knowledge, such a result cannot be shown by any other technique.

\section{VIII.3 Illustration of Theorem 5}

We revisit the unstable second-order linear dynamics from [9], which is the special case of the following when the unknown perturbation terms $\varepsilon(t), \delta_{A}$, and $\delta_{B}$ are all zero functions:

$$
\dot{x}(t)=\left(\left[\begin{array}{cc}
0 & 1 \\
-1 & 1
\end{array}\right]+\delta_{A}(t)\right) x(t)+\left(\left[\begin{array}{l}
0 \\
1
\end{array}\right]+\delta_{B}(t)\right) u(t-h(t))+\varepsilon(t) .
$$

We only apply Theorem 5 and therefore assume that $h \in C^{1}$ is bounded by some constant $g>0$, but we can also apply Theorem 4 to cover cases where the delay can have discontinuities and be uncertain. Choosing

$$
A=\left[\begin{array}{cc}
0 & 1 \\
-1 & 1
\end{array}\right], B=\left[\begin{array}{l}
0 \\
1
\end{array}\right], \text { and } K=-\left[\begin{array}{ll}
2 & 3
\end{array}\right]
$$


the corresponding matrix $M=A+B K$ is Hurwitz, so Assumption 8 is satisfied with $n=2$ and $m=1$. In fact, with the choices

$$
P=\left[\begin{array}{cc}
\frac{4}{3} & \frac{1}{6} \\
\frac{1}{6} & \frac{1}{3}
\end{array}\right] \text { and } c=0.735089
$$

we can satisfy $P M+M^{\top} P \leq-c P$.

To check Assumption 9 and apply Theorem 5, we must construct the required matrices $A_{f}, B_{f}$, and $Q$. To this end, we use the construction from Proposition 1 for the choices (108) and the special case where the tuning parameter is $\nu=1$, but we can take any constant $\nu>0$ and other choices of $P$. With the preceding choices, we get $A_{f}=K B-\frac{c}{2} I_{m}=-3.3675, B_{f}=K A-\frac{2}{\nu} B^{\top} P+\frac{c}{2} K=(1.9316,-6.7693)$, and

$$
Q=\left[\begin{array}{ccc}
10 / 3 & 19 / 6 & 1 \\
19 / 6 & 29 / 6 & 3 / 2 \\
1 & 3 / 2 & 1 / 2
\end{array}\right]
$$

Also, using the fact that $|B|=1$ and lengthy but relatively simple calculations, one checks that Assumptions 8-9 will be satisfied provided there are also constants $\delta \in(0,1)$ and $T>0$ such that

$$
\int_{t-T}^{t}|\dot{h}(\ell)| \mathrm{d} \ell \leq \frac{\delta \sqrt{\lambda_{\min }(Q) / \lambda_{\max }(Q)}-e^{-T c / 2}-\mathcal{M}_{*}(t)}{|A|+2 e^{|A| g}-1}
$$

holds for all $t \geq T$, where

$$
\mathcal{M}_{*}(t)=e^{|A| g}\left(\left(1+\frac{1}{|A|}\left(e^{|A| g}-1\right)\right) e^{|A| g} \int_{t-T}^{t}\left|\delta_{A}(r)\right| \mathrm{d} r+\int_{t-T}^{t}\left|\delta_{B}(r)\right| \mathrm{d} r\right) .
$$

This provides a notable alternative to the corresponding example in [9], since it allows us to conclude input-tostate stability under additive uncertainties $\varepsilon$ (which were not considered in [9]) using the alternative controller from our Theorem 5 (which is based on a dynamic extension that was also not considered in [9]), because we allow additive uncertainty on the coefficient matrices, and because we include the degree of freedom $\nu>0$.

\section{Concluding Remarks}

Time-varying delays can model many important phenomena, such as increasing latencies as a muscle becomes fatigued. Also, sampling in controllers can be modeled using a piecewise $C^{1}$ time-varying delay $h(t)$ where $\dot{h}(t)=1$ almost everywhere. This paper provided new stability techniques for piecewise continuous timevarying linear systems with time-varying delays, and with uncertain coefficient matrices. They can be applied when stabilizing control laws must be designed under delays. Since we also do not require the standard condition $\dot{h}(t)<1$, our results are much broader in scope than much of the existing literature. Two of our theorems use dynamical extensions, including novel results on the reduction method under uncertain delays. Our work uses a trajectory-based method from [35], which circumvents the search for Lyapunov-Krasovskii functionals or small-gain conditions.

The results in this paper are beyond the scope of [35], e.g., since we allow $\sup _{t} h(t)$ to be arbitrarily large, and since our new integral conditions make it possible to establish exponential stability. Also, there are many degrees of freedom in applying our methods (such as a parameter $\nu>0$ in our dynamic extension). In our future work, we hope to exploit the degrees of freedom to make our work applicable to the broadest possible class of systems and chattering delays. Many other extensions seem possible. For instance, we conjecture that our results can be extended to cover distributed delays and systems that are nonlinear in the state, including applications to networked systems with uncertainty. It would also be useful to seek generalizations to hyperbolic PDEs, such as the dynamics for age structured chemostats where the control enters into the PDE directly instead of at the boundary $[23,24]$ and where there may also be state dependent or rapidly varying delays $[4,5,13]$.

\section{References}

[1] Z. Artstein. Linear systems with delayed controls: a reduction. IEEE Transactions on Automatic Control 27(4):869-879, 1982. 
[2] N. Bekiaris-Liberis and M. Krstic. Nonlinear Control under Nonconstant Delays. Society for Industrial and Applied Mathematics, Philadelphia, PA, 2013.

[3] N. Bekiaris-Liberis and M. Krstic. Nonlinear control under delays that depend on delayed states. European Journal of Control 19(5):389-398, 2013.

[4] N. Bekiaris-Liberis and M. Krstic. Compensation of state-dependent input delay for nonlinear systems. IEEE Transactions on Automatic Control 58(2):275-289, 2013.

[5] N. Bekiaris-Liberis and M. Krstic. Robustness of nonlinear predictor feedback laws to time- and statedependent delay perturbations. Automatica 49(6):1576-1590, 2013.

[6] M. Belem Saldivar Marquez, I. Boussaada, H. Mounier, and S-I. Niculescu. Analysis and Control of Oilwell Drilling Vibrations: A Time-Delay Systems Approach. Springer, New York, 2015.

[7] D. Bresch-Pietri, J. Chauvin, and N. Petit. Adaptive control scheme for uncertain time-delay systems. Automatica 48(8):1536-1552, 2012.

[8] D. Bresch-Pietri, J. Chauvin, and N. Petit. Prediction-based stabilization of linear systems subject to input-dependent input delay of integral-type. IEEE Transactions on Automatic Control 59(9):2385-2399, 2014.

[9] D. Bresch-Pietri and N. Petit. Robust compensation of a chattering time-varying input delay. In Proceedings of the IEEE Conference on Decision and Control, Los Angeles, CA, 2014, pp. 457-462.

[10] J.-Y. Dieulot and J-P. Richard. Tracking control of a nonlinear system with input-dependent delay. In Proceedings of the 40th IEEE Conference on Decision and Control, Orlando, FL, 2001, pp. 4027-4031.

[11] R. Downey, R. Kamalapurkar, N. Fischer, and W. Dixon. Compensating for fatigue-induced time-varying delayed muscle response in neuromuscular electrical stimulation control. In Recent Results on Nonlinear Time Delayed Systems. Springer, New York, 2015, pp. 143-161.

[12] L. Figueredo, J. Ishihara, G. Borges, and A. Bauchspiess. Robust stability criteria for uncertain systems with delay and its derivative varying within intervals. In Proceedings of the American Control Conference, San Francisco, CA, 2011, pp. 4884-4889.

[13] E. Fridman and S-I. Niculescu. On complete Lyapunov-Krasovskii functional techniques for uncertain systems with fast-varying delays. International Journal of Robust and Nonlinear Control 18(3):364-374, 2008.

[14] E. Fridman, A. Seuret, and J-P. Richard. Robust sampled-data stabilization of linear systems: An input delay approach. Automatica 40(8):1441-1446, 2004.

[15] E. Fridman and U. Shaked. Delay-dependent stability and $H_{\infty}$ control: constant and time-varying delays. International Journal of Control 76(1):48-60, 2003.

[16] H. Gao, T. Chen, and J. Lam. A new delay system approach to network-based control. Automatica 44(1):39$52,2008$.

[17] K. Gu, V. Kharitonov, and J. Chen, Stability of Time-Delay Systems. Birkhauser, Boston, 2003.

[18] I. Haidar, P. Mason, S-I. Niculescu, M. Sigalotti, and A. Chaillet. Further remarks on Markus-Yamabe instability for time-varying delay differential equations. In Proceedings of the 12th IFAC Workshop on Time Delay Systems, Ann Arbor, MI, 2015, pp. 33-38.

[19] A. Halanay. Differential Equations: Stability, Oscillations, Time Lags. Academic Press, New York, 1966.

[20] J. Hale. Ordinary Differential Equations. Second Edition Robert E. Krieger Publishing Company, Malabar, FL, 1980.

[21] A. Ivanov, E. Liz, and S. Trofimchuk. Halanay inequality, Yorke 3/2 stability criterion, and differential equations with maxima. Tohoku Mathematical Journal 54(2):277-295, 2002.

[22] M. Jankovic. Recursive predictor design for linear systems with time delay. In Proceedings of the American Control Conference, Seattle, WA, 2008, pp. 4904-4909.

[23] I. Karafyllis and M. Krstic. On the relation of delay equations to first-order hyperbolic partial differential equations. ESAIM Control, Optimisation and Calculus of Variations 20(3):894-923, 2014. 
[24] I. Karafyllis, M. Malisoff, and M. Krstic. Sampled-data feedback stabilization of age-structured chemostat models. In Proceedings of the 2015 American Control Conference, Chicago, IL, 2015, pp. 4549-4554.

[25] H. Khalil. Nonlinear Systems, Third Edition. Prentice Hall, Englewood Cliffs, NJ, 2002.

[26] V. Kharitonov and S-I. Niculescu. On the stability of linear systems with uncertain delay. IEEE Transactions on Automatic Control 48(1):127-132, 2002.

[27] M. Krstic. Delay Compensation for Nonlinear, Adaptive, and PDE Systems. Birkhauser, Boston, 2009.

[28] M. Krstic. Lyapunov stability of linear predictor feedback for time-varying input delay. IEEE Transactions on Automatic Control 55(2):554-559, 2010.

[29] W. Kwon and A. Pearson. Feedback stabilization of linear systems with delayed control. IEEE Transactions on Automatic Control 25(2):266-269, 1980.

[30] K. Liu, V. Suplin, and E. Fridman. Stability of linear systems with general sawtooth delay. IMA Journal of Mathematical Control and Information 27(4):419-436, 2010.

[31] J. Louisell. New examples of quenching in delay differential equations having time-varying delay. In Proceedings of the 5th European Control Conference, Karlsruhe, Germany, 1999.

[32] A. Manitius and A. Olbrot. Finite spectrum assignment problem for systems with delays. IEEE Transactions on Automatic Control 24(4):541-552, 1979.

[33] L. Markus and H. Yamabe. Global stability criteria for differential systems. Osaka Mathematical Journal 12(2):305-317, 1960.

[34] F. Mazenc and M. Malisoff. New technique for stability analysis for time-varying systems with delay. In Proceedings of the IEEE Conference on Decision and Control, Los Angeles, CA, 2014, pp. 1215-1220.

[35] F. Mazenc and M. Malisoff. Trajectory based approach for the stability analysis of nonlinear systems with time delays. IEEE Transactions on Automatic Control 60(6):1716-1721, 2015.

[36] F. Mazenc and M. Malisoff. Reduction model approach for systems with a time-varying delay. In Proceedings of the IEEE 54th Conference on Decision and Control, Osaka, Japan, 2015, pp. 7723-7727.

[37] F. Mazenc, M. Malisoff, and T. Dinh. Robustness of nonlinear systems with respect to delay and sampling of the controls. Automatica 49(6):1925-1931, 2013.

[38] F. Mazenc, M. Malisoff, and Z. Lin. Further results on input-to-state stability for nonlinear systems with delayed feedbacks. Automatica 44(9):2415-2421, 2008.

[39] F. Mazenc, M. Malisoff, and S-I. Niculescu. Reduction model approach for linear time-varying systems with delays. IEEE Transactions on Automatic Control 59(8):2068-2082, 2014.

[40] F. Mazenc, M. Malisoff, and S-I. Niculescu. Stability analysis for systems with time-varying delay: trajectory based approach. In Proceedings of the IEEE 54th Conference on Decision and Control, Osaka, Japan, 2015, pp. 1811-1816.

[41] F. Mazenc, S-I. Niculescu, and M. Krstic. Lyapunov-Krasovskii functionals and application to input delay compensation for linear time-invariant systems. Automatica 48(7):1317-1323, 2012.

[42] W. Michiels and S-I. Niculescu. Stability and Stabilization of Time-Delay Systems. Society for Industrial and Applied Mathematics, Philadelphia, PA, 2007.

[43] S. Mondié and W. Michiels. Finite spectrum assignment of unstable time-delay system with a safe implementation. IEEE Transactions on Automatic Control 48(12):2207-2212, 2003.

[44] N. Petit, Y. Creff, and P. Rouchon. Motion planning for two classes of nonlinear systems with delays depending on the control. In Proceedings of the 37th IEEE Conference on Decision and Control, Tampa, FL, 1998, pp. 1007-1011.

[45] J-P. Richard. Time-delay systems: an overview of some recent advances and open problems. Automatica 39(10):1667-1694, 2003.

[46] W. Rudin. Principles of Mathematical Analysis, Third Edition. McGraw-Hill, New York, 1976.

[47] M. Sbarciog, R. De Keyser, S. Cristea, and C. De Prada. Nonlinear predictive control of processes with variable time delay. A temperature control case study. In Proceedings of the IEEE International Conference on Control Applications, San Antonio, TX, 2008, pp. 1001-1006.

[48] O. Smith. A controller to overcome dead time. ISA Journal, 6(2):28-33, 1959.

[49] B. Zhou. Truncated Predictor Feedback for Time-Delay Systems. Springer, London, UK, 2014. 